\title{
RELATING NATURALISTIC GLOBAL POSITIONING SYSTEM (GPS) DRIVING DATA WITH LONG-TERM SAFETY PERFORMANCE OF ROADWAYS
}

\author{
A Thesis \\ presented to \\ the Faculty of California Polytechnic State University, \\ San Luis Obispo
}

\author{
In Partial Fulfillment \\ of the Requirements for the Degree \\ Master of Science in Civil and Environmental Engineering \\ by \\ James Michael Loy
}

August 2013 
(C) 2013

James Michael Loy

\section{ALL RIGHTS RESERVED}




\section{COMMITTEE MEMBERSHIP}

TITLE:

Relating Naturalistic Global Positioning System (GPS) Driving Data with Long-Term Safety Performance of Roadways

AUTHOR: James Michael Loy

DATE SUBMITTED: $\quad$ August 2013

COMMITTEE CHAIR: $\quad$ Anurag Pande, Ph.D.

Assistant Professor

Department of Civil and Environmental

Engineering

COMMITTEE MEMBER: Kimberley Mastako, Ph.D.

Lecturer

Department of Civil and Environmental

Engineering

COMMITTEE MEMBER: $\quad$ Brian Wolshon, Ph.D., P.E.

Professor

Civil and Environmental Department

Louisiana State University 


\section{ABSTRACT \\ Relating Naturalistic Global Positioning System (GPS) Driving Data with Long-Term Safety Performance of Roadways James Michael Loy}

This thesis describes a research study relating naturalistic Global Positioning System (GPS) driving data with long-term traffic safety performance for two classes of roadways. These two classes are multilane arterial streets and limited access highways. GPS driving data used for this study was collected from 33 volunteer drivers from July 2012 to March 2013. The GPS devices used were custom GPS data loggers capable of recording speed, position, and other attributes at an average rate of 2.5 hertz.

Linear Referencing in ESRI ArcMAP was performed to assign spatial and other roadway attributes to each GPS data point collected. GPS data was filtered to exclude data with high horizontal dilution of precision (HDOP), incorrect heading attributes or other GPS communication errors.

For analysis of arterial roadways, the Two-Fluid model parameters were chosen as the measure for long-term traffic safety analysis. The Two-Fluid model was selected based on previous research which showed correlation between the Two-Fluid model parameters $n$ and $T_{m}$ and total crash rate along arterial roadways. Linearly referenced GPS data was utilized to obtain the total travel time and stop time for several half-mile long trips along two arterial roadways, Grand Avenue and California Boulevard, in San Luis Obispo. Regression between log transformed values of these variables (total travel time and stop time) were used to derive the parameters $n$ and $T_{m}$. To estimate stop time for each trip, a vehicle "stop" was defined when the device was traveling at less than 2 miles per hour. Results showed that Grand Avenue had a higher value for $n$ and a lower value for $T_{m}$, which suggests that Grand Avenue may have worse long-term safety performance as characterized by long-term crash rates. However, this was not verified with crash data due to incomplete crash data in the TIMS database. Analysis of arterial roadways concluded by verifying GPS data collected in the California Boulevard study with sample data collected utilizing a traditional "car chase" methodology, which showed that no significant difference in the two data sources existed when trips included noticeable stop times.

For analysis of highways the derived measurement of vehicle jerk, or rate of change of acceleration, was calculated to explore its relationship with long-term traffic safety performance of highway segments. The decision to use jerk comes from previous research which utilized high magnitude jerk events as crash surrogate, or near-crash events. Instead of using jerk for near-crash analysis, the measurement of jerk was utilized to determine the percentage of GPS data observed below a certain negative jerk threshold for several highway segments. These segments were $1 / 4$-mile and $1 / 2$-mile long. The preliminary exploration was conducted with 39 1/4-mile long segments of US Highway 101 within the city limits of San Luis Obispo. First, Pearson's correlation coefficients were estimated for 
rate of 'high' jerk occurrences on these highway segments (with definitions of 'high' depending on varying jerk thresholds) and an estimate of crash rates based on long-term historical crash data. The trends in the correlation coefficients as the thresholds were varied led to conducting further analysis based on a jerk threshold of $-2 \mathrm{ft}$./ $/ \mathrm{sec}^{3}$ for the $1 / 4$-mile segment analysis and $-1 \mathrm{ft} . / \mathrm{sec}^{3}$ for the $1 / 4$-mile segment analysis. Through a negative binomial regression model, it was shown that utilizing the derived jerk percentage measure showed a significant correlation with the total number of historical crashes observed along US Highway 101. Analysis also showed that other characteristics of the roadway, including presences of a curve, presence of weaving (indicated by the presence of auxiliary lanes), and average daily traffic (ADT) did not have a significant correlation with observed crashes. Similar analysis was repeated for 19 1/2-mile long segments in the same study area, and it was found the percentage of high negative jerk metric was again significant with historical crashes. The 1/2-mile negative binomial regression for the presence of curve was also a significant variable; however the standard error for this determination was very high due to a low sample size of analysis segments that did not contain curves.

Results of this research show the potential benefit that naturalistic GPS driving data can provide for long-term traffic safety analysis, even if data is unaccompanied with any additional data (such as live video feed) collected with expensive vehicle instrumentation. The methodologies of this study are repeatable with many GPS devices found in certain consumer electronics, including many newer smartphones.

Keywords: Naturalistic driving, Two-Fluid model, network performance, driver behavior, traffic safety, global positioning systems 


\section{ACKNOWLEDGMENTS}

I would like to thank my graduate advisor, Dr. Anurag Pande for his work on this project and for serving as chair of my graduate committee. Dr. Pande's experience in data mining, traffic models, and statistical analysis software proved to be very beneficial for this project. In addition to Dr. Pande, I would also like to thank the other two individuals on my thesis committee, Dr. Kimberley Mastako and Dr. Brian Wolshon. Dr. Mastako has had a very strong influence on my education at Cal Poly, and I thank her not only for serving on this committee, but also for helping me progress during my undergraduate and graduate career. Dr. Wolshon of Louisiana State University deserves a very large acknowledgment for his help with this project and the excellent advice he has given me for this thesis and the conference papers we have submitted together.

I would also like to thank the many others that have contributed to this project. This includes Dr. Vinayak Dixit of the University of New South Wales for his help with data processing and validation relating to the Two-Fluid model. Katherine Spansel of Louisiana State University also deserves acknowledgement for her work in research and methodology. In addition, acknowledgements also go to both Russell White at Cal Poly's GIS Research Center and Dr. Josh Kent at the Center for Geolnformatics at Louisiana State University for their technical contributions and guidance on the application of ArcMap for this research. Sean Carney and Nathan Johnston were also indispensable for their aid in collecting GPS data and Two-Fluid data in San Luis Obispo. Also, although they must remain unnamed, I would like to thank all the volunteer drivers in this study. Finally, I must thank the National Science Foundation (NSF) for their monetary support of this research.

On a personal note, I would also like to thank my family, friends, and colleagues for helping me and supporting me during school. Your support and generosity far exceeded anything than I ever expected, and I will forever be grateful for all the aid I have received. 


\section{TABLE OF CONTENTS}

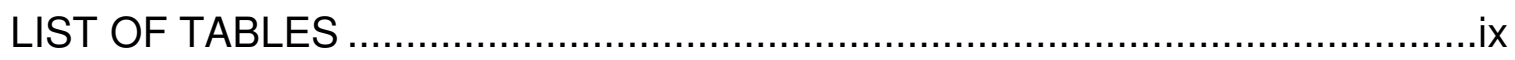

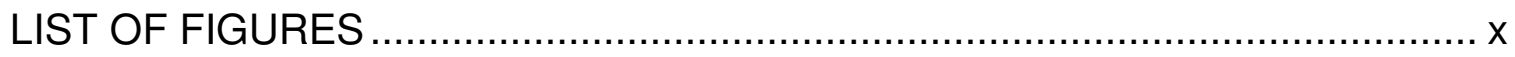

CHAPTER

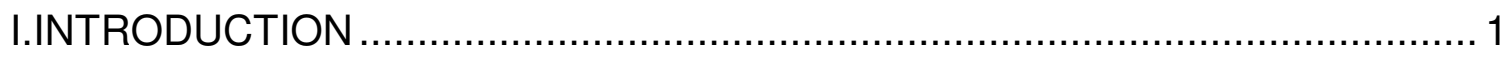

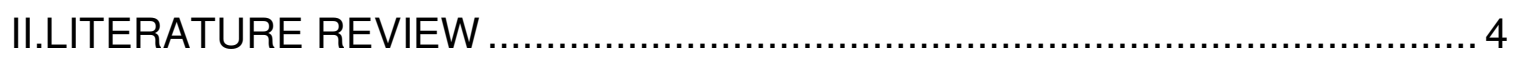

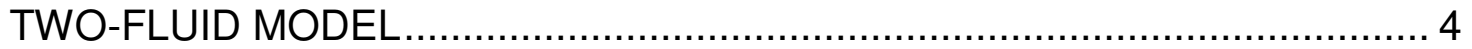

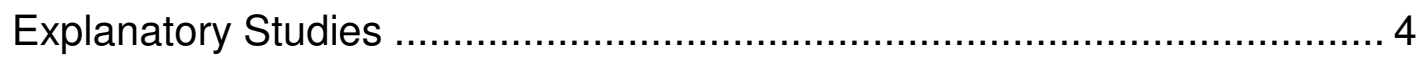

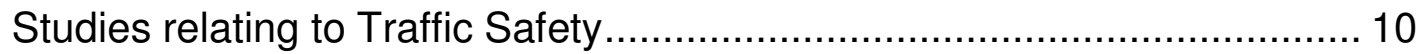

Studies utilizing GPS Data ............................................................. 10

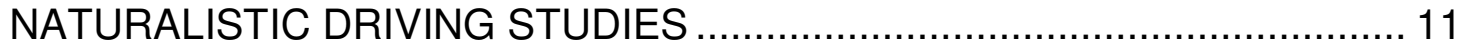

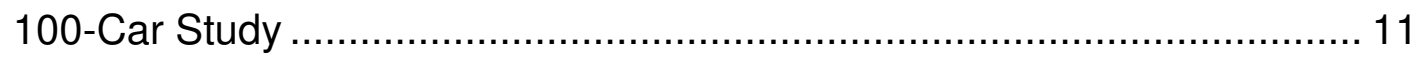

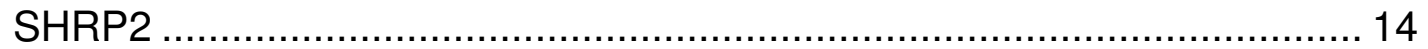

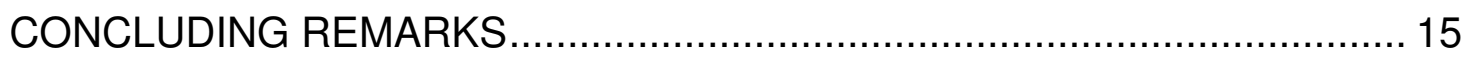

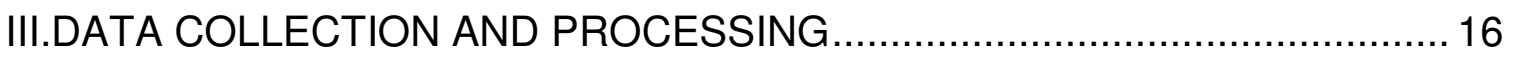

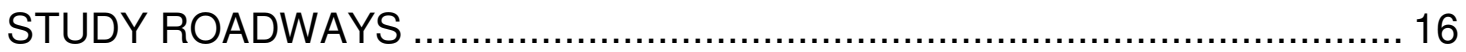

DATA COLLECTION AND PREPARATION .................................................. 18

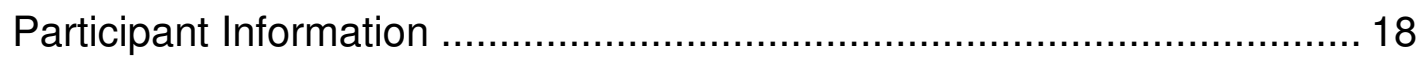

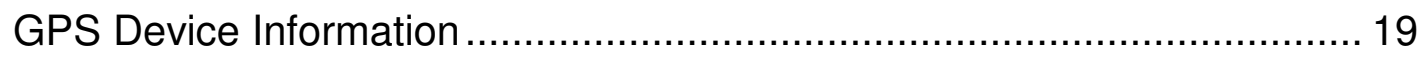

GPS Data Collection Process and Associated Errors ............................... 22

Data Processing and GIS Linear Referencing ......................................... 24

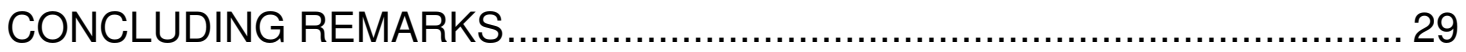

IV. TWO-FLUID MODEL ANALYSIS ....................................................... 30

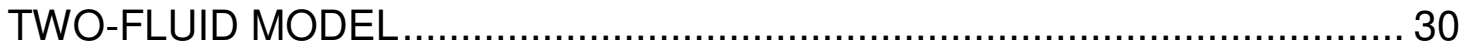

DETERMINATION OF TWO-FLUID PARAMETERS …............................... 32

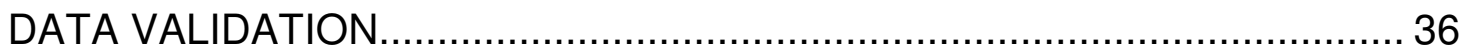

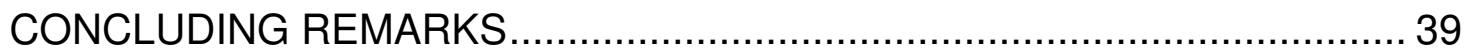

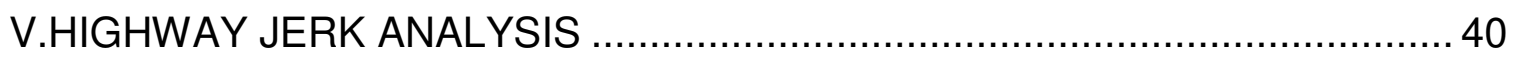

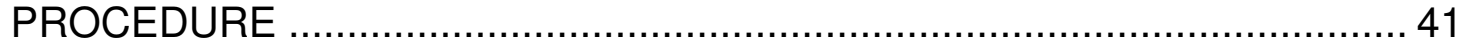

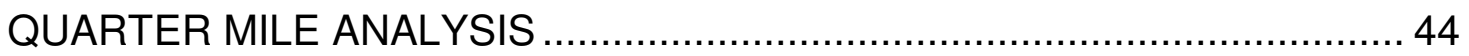




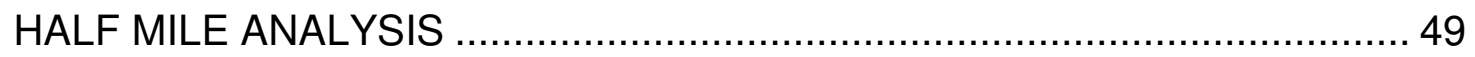

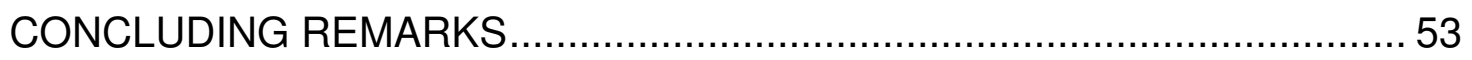

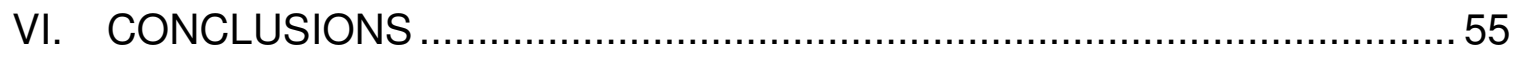

TWO-FLUID ANALYSIS FOR ARTERIAL ROADWAYS ….......................... 55

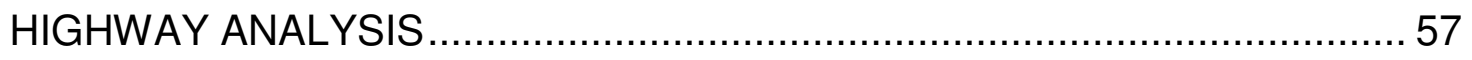

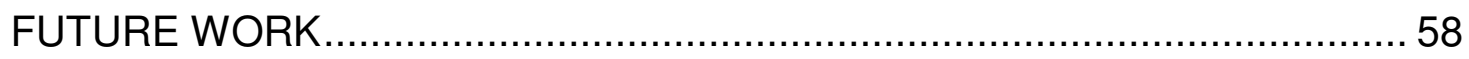

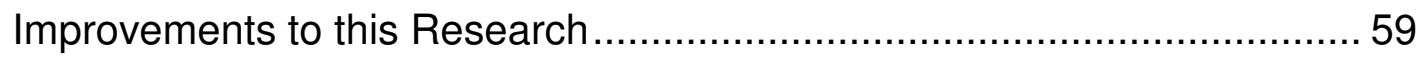

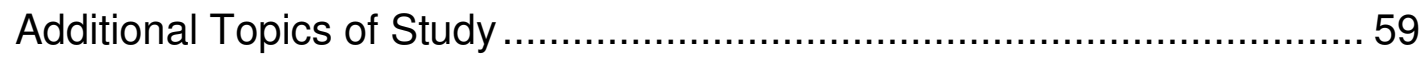

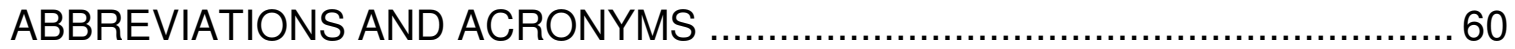

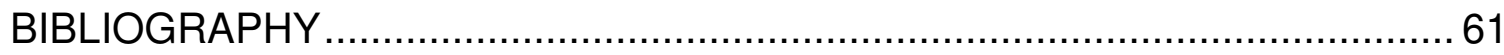

APPENDICES

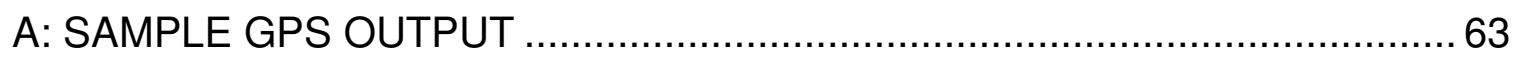

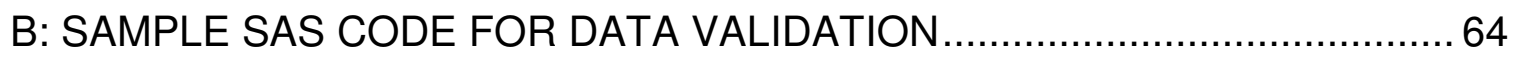

C: SAMPLE SAS CODE FOR HIGHWAY ANALYSIS ..................................... 71 


\section{LIST OF TABLES}

Table 1. Two-Fluid Model Parameters in Literature ........................................... 7

Table 2. Sign of Correlation between Network Features and Two-Fluid

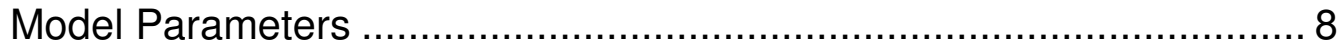

Table 3. GPS Data Logger Attributes Recorded.......................................... 21

Table 4. Two-Fluid Parameters Obtained ........................................................ 34

Table 5. Linear Model Data Verification Results............................................ 38

Table 6. Crash Estimation utilizing Negative Binomial Regression Models for Quarter Mile Segments .................................................................. 47

Table 7. Crash Estimation utilizing Negative Binomial Regression Models for Half Mile Segments........................................................................... 51 


\section{LIST OF FIGURES}

Figure 1. Arterial Segments of Interest: Grand Avenue and California

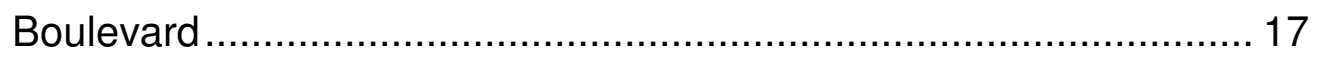

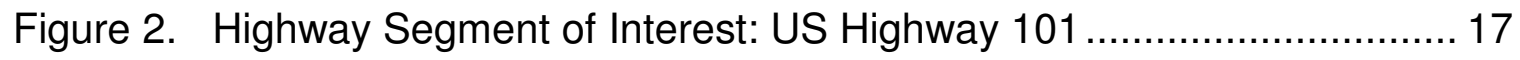

Figure 3. Histogram of Participant Residences............................................ 19

Figure 4. OHARARP SD GPS DataLogger V.315 .................................... 20

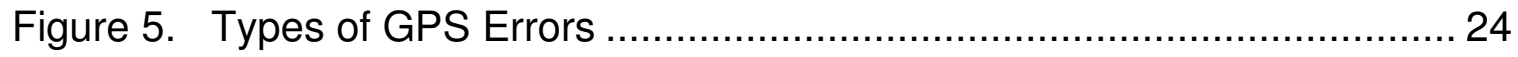

Figure 6. San Luis Obispo County GIS Roadway Base Map........................... 25

Figure 7. Linear Referencing and Dynamic Segmentation Example ............... 28

Figure 8. Relation between Total Travel Time and Stop Time for $1 / 2-$ Mile

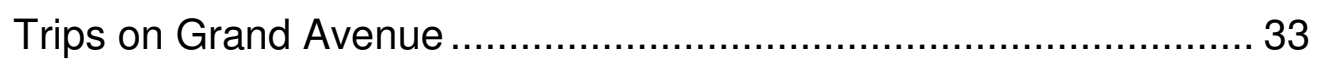

Figure 9. Relation between Total Travel Time and Stop Time for 1/2-Mile Trips on California Boulevard ......................................................... 34

Figure 10. Relation between Total Travel Time and Stop Time for $1 / 2-$ Mile Trips on California Boulevard with GPS Device Data and Car Chase Data ............................................................................. 37

Figure 11. Pearson's Correlation Coefficient for varying jerk thresholds ........... 43

Figure 12. Straight line diagrams for US NB (left, 1-20) and SB (right, 21-39)

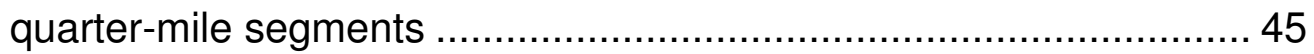

Figure 13. Linear Heat Maps for Quarter Mile Analysis.................................... 49

Figure 14. Straight line diagrams for US NB (left, 1-10) and SB (right, 11-20)

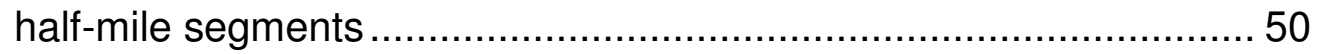

Figure 15. Linear Heat Maps for Half Mile Analysis ....................................... 53 


\section{INTRODUCTION}

While transportation engineers design roadways to meet specific standards for traffic safety, certain roadway conditions still contribute to the rise of unsafe locations for drivers. For many agencies, the traditional method to identify these unsafe locations relies on historical traffic crash data. Using the measure of traffic crashes, however, has the disadvantage of taking long periods of time to collect before any trends in traffic safety can be made.

Recent research efforts, however, have sought to identify better and more efficient methods to identify hazardous driving conditions and locations. The utilization of detailed naturalistic driving data, such as the 100-Car Study performed by the Virginia Tech Transportation Institute (Neale, 2005), has begun to shift research towards searching for abnormal driving events that cause near-collisions or events that have the potential to cause a vehicle collision. These events, otherwise known as crash surrogates or crash conflicts, have the potential to strengthen traditional traffic safety analysis methods by identifying areas of high crash potential before crashes actually occur (Hauer, 1996). Crash surrogate events are also advantageous in the regard that they are more common; meaning that analysis with crash surrogates can be performed over significantly shorter durations of time.

The concept of crash surrogates is easy to comprehend but it often harder to identify these events within larger data sources. Many naturalistic driving studies, such as the 100-Car Study, utilize high-end video cameras and motion sensors that require detailed analysis and are impractical to recommend for large scale 
studies over multiple areas.

These naturalistic driving studies performed for the identification of surrogate events can help researchers to understand various attributes of traffic incidents. However, naturalistic driving studies can also provide other valuable information for a variety of other transportation models and studies. This includes research in the field of long-term traffic safety analysis. This study focused on collecting and utilizing naturalistic driving data obtained from Global Positioning System (GPS) devices for the purpose of improving traditional traffic safety methodologies for various types of transportation facilities, including arterial roadways and highways. The research associated with this study used the following steps:

1. Conduct preliminary literature review to determine appropriate parameters that indicate long-term traffic safety performance for highway and arterial roadways.

2. Enlist participants to serve as volunteer drivers via an online screening survey that solicits personal and driving information. Cluster individuals into groups and select top candidates based on driving frequency and other factors.

3. Select a GPS device capable of recording driving data at a rate suitable for analysis.

4. Collect GPS driving data from selected participants over two-week periods with GPS devices. Assemble a complete data set with driving data from selected participants and assign each driver a random iden- 
tification code to assure participant confidentiality.

5. Process and filter the GPS data in ArcMap by identifying and removing erroneous data. Utilize linear referencing in ArcMap to match attributes of GPS driving data with set attributes of traveled roadways.

6. For each trip on selected arterial roadways, employ ArcMap to obtain Two-Fluid model parameters from travel time and stop time. Comment on the results of the analysis and the validity of this data for Two-Fluid model analysis.

7. Determine the best measurement to use for long-term traffic safety analysis on highway segments with GPS data.

8. Compare trends seen in measurements from GPS driving data and historical crash rates to determine if statistical links exists.

This thesis is organized into six chapters, including this introductory Chapter 1. The following chapter, Chapter 2, provides a literature review of naturalistic driving studies and previous research findings. Chapter 3 describes the data collection and processing methodologies of this research, including specific information about the GPS devices and the cleaning methods utilized. Chapter 4 discusses the specific results for arterial roadways including the parameters of the TwoFluid model. Chapter 5 describes the methodology and results of examining the rate of occurrence of high jerk (the rate of change of acceleration) as a measure on highway segments. Chapter 6 concludes the research and suggests future research topics relating to the GPS data and the research methods of this study. 


\section{LITERATURE REVIEW}

This chapter reviews previous studies from the literature relevant to this research. The literature review is divided into two sections. The first section is a summary the Two-Fluid model, which is a traffic flow model utilized for analysis of arterial roadways in this study. This section also includes previous findings and research relating the Two-Fluid model to traffic safety performance. The second section is a discussion of recent naturalistic driving studies, and some of the corresponding literature that has been published relating to traffic safety. This literature review shows previous work performed related to this study and also identify gaps in the literature that exist.

\section{TWO-FLUID MODEL}

\section{Explanatory Studies}

Herman and Prigogine formally introduced the Two-Fluid model in 1979 as a method that could quantify the quality of traffic flow on urban traffic networks. (Williams, 1996) The model is a macroscopic flow model that views traffic flow in a network as a collection of vehicles in one of two states, or fluids; stopped or running. The development of the Two-Fluid model was an extension of previous work formulated during earlier vehicle kinematic theories for multiple lane traffic performed by Herman and Prigogine (1971). To derive the Two-Fluid model, researchers investigated a wide variety of parameters relating to vehicle flow within a network, including average speed, stopped time, and speed distribution functions, from previous data collected data for multiple cities. It was consistently observed that the variables of travel time per unit and stopped time per unit in the 
network (caused by traffic jams, stop lights) had a positive linear relationship where total travel time increased as a linear function of stopped time. Herman and Prigogine first verified the linear relationship observed in each data set by finding the values of the two linear coefficients, $A$ and $B$, and a correlation coefficient for each data set. Results of the analysis showed that the estimates for $A$ and $B$ varied for different data sets but the correlation coefficients remained high in each dataset.

Using two assumptions about traffic flow, Herman and Prigogine then discussed the possibility of two parameters that emerged from the data that could be used for analysis of traffic networks, $n$ and $T_{m}$. The first assumption came from their prior work in which they stated that the average running speed in a street network was proportional to the fraction of vehicles moving in it. The second assumption was stated that the fractional stop time of a test vehicle circulation in a network was equal to the average fraction of the vehicles stopped during the same period. At the time of writing, Herman and Prigogine could not verify the second assumption but later work (Ardekani and Herman, 1987) helped to verify the assumptions and made the Two-Fluid model valid.

The parameters $n$ and $T_{m}$ were determined by Herman and Prigogine to represent qualities of the network. The parameter $T_{m}$ designated the average minimum travel trip time in a given network. Therefore this $T_{m}$ value represented the average time among multiple trips in which no traffic or stopping was encountered in the network. The parameter $n$ was perceived to be a measure of how susceptible a particular network was to traffic congestion due to increased vehi- 
cle demand. The linear interpretation of the Two-Fluid parameters is that the parameter $n$ represents the slope of a trend line plotted on a time stopped versus time travel plot, and that $T_{m}$ represents the y-axis intercept. Based on this linear definition, the value of $n$ must be greater than zero and $T_{m}$ must be a positive value. (Herman and Prigogine, 1979)

The data collection method utilized by Herman and Prigogine and other data sources was collected using a "car chase" methodology. In this, researchers followed randomly selected subject vehicles within a defined network of streets. With this car chase methodology, researches were instructed follow to a randomly selected vehicle until that vehicle parked, drove out of the subject area, or performed an unsafe maneuver. During the chase, observations on total travel time per unit measure, total running time per unit measure, and total stopped time per unit measure were recorded. The typical unit of measure for initial Two-Fluid testing ranged from 1 kilometer to 2 miles. Typically this procedure required two researchers in the chase vehicle.

Since the development of the Two-Fluid model, many other studies have been have been conducted in various cities to show that the Two-Fluid model parameters can be used to characterize urban street networks (Herman and Ardekani, 1984; Ardekani and Herman 1987; Ardekani et al. 1985). Table 1 created by Lee et al. (2005) summarized the results obtained from the Two-Fluid parameters from various studies conducted by various researches over the past 30 years. 
Table 1. Two-Fluid Model Parameters in Literature

\begin{tabular}{|l|c|c|c|}
\hline City & $T_{m}(\mathrm{~min} / \mathrm{mile})$ & $n$ & $R^{2}$ \\
\hline Austin & 1.78 & 1.65 & 0.78 \\
\hline Dallas & 1.97 & 1.48 & 0.80 \\
\hline Houston & 2.70 & 0.80 & 0.63 \\
\hline San Antonio & 2.01 & 1.49 & 0.84 \\
\hline Milwaukee & 1.59 & 1.41 & 0.81 \\
\hline London & 1.93 & 3.02 & 0.97 \\
\hline Melbourne & 1.74 & 1.41 & 0.95 \\
\hline Sydney & 1.85 & 1.68 & 0.88 \\
\hline Brussels & 1.26 & 2.76 & 0.92 \\
\hline Seoul Kangnam & 2.17 & 0.90 & 0.69 \\
\hline
\end{tabular}

Source: Lee et al. (2005)

While the Two-Fluid model is a popular model based on its ease of implementation and use, the parameters $T_{m}$ and $n$ can be sometimes overlooked in analysis. A large portion of research relating to the Two-Fluid model has been dedicated to further understand the various attributes that affect the model and to what extent the parameters can be impacted. Herman et al. (1988) aimed to investigate the impact that extreme driver behavior had on the results of the Two-Fluid parameters obtained. As part of this study, the traditional car chase methodology was utilized within the cities of Austin, Texas, and Roanoke, Virginia. With this research, however, multiple car chase periods were performed with varying instructions pertaining to how aggressively the researchers should drive. Three car chase runs were performed for each network, one collection period with normal driving, one period with conservative driving and one period with aggressive driving. In theory, more aggressive driving would result in lower values for $T_{m}$. Re- 
sults of the analysis showed that driver aggressiveness does have a significant impact on Two-Fluid model obtained. From the Austin dataset it was also observed that the linear trend lines in aggressive and conservative driving tend to converge at a single point with a high stopped time. This suggests that the $n$ value for aggressive driving was higher compared to conservative driving behavior.

Other research has studied effects of the Two-Fluid parameters caused by geometric and operational differences in networks. Table 1 compiled by Dixit et al. (2011) summarizes the various geometric and operational differences between various networks, and their respective correlation with the Two-Fluid model parameters $n$ and $T_{m}$.

Table 2. Sign of Correlation between Network Features and Two-Fluid Model Parameters

\begin{tabular}{|l|c|c|}
\hline Factor & $T_{m}$ & $n$ \\
\hline Signal Density & + & - \\
\hline Average Speed Limit & - & - \\
\hline Fraction of approaches with signal progression & - & \\
\hline Average number of lanes per street & - & - \\
\hline Fraction of one way streets & + & + \\
\hline Fraction with actuated signals & & + \\
\hline Average block lengths & & + \\
\hline Average cycle length & - & + \\
\hline
\end{tabular}

Source: Dixit et al. (2011)

Lee et al. (2005) sought to investigate the impact that weather changes would have on Two-Fluid model parameters. To investigate this impact, researches collected Two-Fluid model in the metropolitan area of Seoul, South Korea before and after a snowing event occurred. Using statistical tests to determine the im- 
pact of the snowing event, researchers found that both $T m$ and $n$ significantly increased after the snowing event and did not return to normal values until three days after the event occurred. Researchers did not perform the analysis for any other type of weather event.

The Two-Fluid model has also been utilized by researchers to perform before and after analysis for particular network areas. One of the more recent studies conducted by Vo et al. (2007) investigated Two-Fluid parameters from calibrated models from two cities from two separate time periods before and after major improvements were made to the network. The results of the analysis differed with each city. Results for the city of Arlington, Texas showed that no significant changes in Two-Fluid parameters were observed despite improvements made to network.

Jones and Farhat (2004) sought to validate the assumption that the Two-Fluid model could be applied for analysis on individual arterial roadways, and not just entire network-wide scale. Utilizing data collected from two arterial roadways in Omaha, Nebraska, researchers proved that the Two-Fluid parameters are effective in assessing the quality of traffic between different arterial streets, over varying time periods on the same arterial roadway, or on separate portions of an arterial street. Researches believed this finding could mean that the Two-Fluid model parameters could possible act as a measure of effectiveness (MOE) to rate various traffic engineering projects. 


\section{Studies relating to Traffic Safety}

Dixit et al. (2011) sought to utilize the traditional Two-Fluid model parameters for the purpose of traffic safety analysis. Utilizing the car chase methodology, researchers collected data to obtain Two-Fluid parameters from 8 arterial roadways in the downtown Orlando, Florida. By calculating crash rates from crash information obtained from the Florida Crash Analysis and Reporting (CAR) database, researches determined the correlation coefficients between the parameters of $n$ and $T_{m}$ with crash rate of varying severity and type. Through the analysis it was found that the parameter $n$ had a positive significant correlation with rear end crash rate and severe crash rate. The parameter $T_{m}$ had a negative significant correlation with rear end crash rate and severe crash rate. Researchers suggest further that while this type of research could be used as a surrogate to help shorten the period required for traffic safety analysis, more research would need to be performed.

\section{Studies utilizing GPS Data}

Hong et al. (2005) sought to test the validity of using higher end GPS equipment for the determination of Two-Fluid parameters. Using GPS equipment that mounted to a vehicle's control panel, researchers drove around the Kangnam network in Seoul, Korea under normal weather conditions. GPS data collected was recorded at a rate of 1 hertz ( 1 reading per second). A cut-off speed of 2 kilometer per hour was implemented by researchers in the data set to represent when the vehicle was stopped. From a small sample size, researchers determined parameters $n$ and $T_{m}$ for 1.5 kilometer segments and compared them with 
previous research in which these values were determined. The values from their previous research and their GPS collected data varied slightly but the results were deemed to be significantly similar. No further analysis utilizing the derived Two-Fluid model parameters was performed by researchers.

\section{NATURALISTIC DRIVING STUDIES}

\section{0-Car Study}

The 100-Car Naturalistic Driving Study was the first instrumented vehicle study undertaken with the primary purpose of collecting large-scale naturalistic driving data. (Neale et al., 2005). The study utilized high end instrumentation custom made by Virginia Tech to be rugged, durable, expandable and un-obstructive to participant drivers. The major component to the instrumentation was the data acquisition system (DAS) which contained a Pentium-based computer and a large hard drive that could store weeks of driving data at a time. The DAS typically was positioned in the rear trunk of the vehicle. Connected to the DAS was a series of sensors that included accelerometers, a headway detection system, side obstacle detection sensors, and Doppler radar sensors installed on the front and rear end of the vehicle. Also connected to the DAS was a set of five video cameras that recorded various perspectives of the vehicle and driver. Through this instrumentation, researchers had a very strong understanding of not only what conditions drivers were exposed to at each instance but also information relating to the state or actions of the driver during while driving.

As the name of the research would suggest, a total of 100 vehicles were 
equipped with the DAS and sensors. The majority of these vehicles (78 out of 100) were personal vehicles of the participants involved in the study. Due to the large amount of custom made brackets that were required to equip vehicles with the necessary instrumentation, only six models of passenger vehicles were seen in the data set. A total of 109 primary drivers were included in this study, and data was also collected from 132 additional drivers who utilized the equipped vehicles during the study period. All primary participants were individuals who commuted into or out of the Northern Virginia / Washington DC area. Drivers were both male and female, and were 18 years and older.

The total 100 -car study recorded approximately $2,000,000$ vehicle miles of data, or the equivalent of 43,000 hours of data. While a total of 83 crashes occurred during the data recording period, only 13 incidents had to be disregarded due to feedback errors. Using a rough definitions of near-crash (a conflict situation requiring a rapid, severe evasive maneuver) and incident (conflict requiring an evasive maneuver but to a lower magnitude), early researchers identified a total of 761 near-crashes and 8295 incidents were present in the complete dataset.

Klauer et al. (2006) utilized the data collected from the 100-car study for further analysis investigating risk associated with varying types of driver inattentiveness. In this analysis, various types of driver distractions and driving conditions were investigated. Utilizing near-crash, crash, and normal driving baseline data various risks associated with different types of driver conditions and actions were calculated. Results showed that drowsiness behind the wheel resulted in a risk four to six time higher relative to drivers who were alert. Results also showed that driv- 
ers engaged in visually or manually complicated tasks (such as distraction from an electronic device) were three times more likely to have a crash or near-crash event occur. Researchers also showed that there are certain environmental conditions in which these factors are more dangerous. This list included intersections, wet roadways, and areas of high traffic density.

Further work by Guo et al. (2010) utilized the data from the 100-car study to determine the validity of determining near-crashes (crash surrogates) within naturalistic driving data for traffic safety analysis. The need to identify crash surrogates from the 100-car study came as a result of the low number of observed accidents seen in the total dataset. In this analysis, a near-crash event was defined as any circumstance that requires rapid, evasive maneuver by the participant's vehicle or any other vehicle, pedestrian, bicyclist, or animal to avoid a crash. (Guo et al. 2010) The observed frequency of the near-crash was in the range of 10 to 15 times more prominent than crash events were. The major portion of the research proved that there was a strong frequency relationship between crash and nearcrash events, and that there was no evidence to suggest that the causal mechanism between crash and near crash varies. Because of these findings, researchers state that within small sample size using near-crashes as surrogates can significantly improve the precision of crash estimation.

Bagdadi and Varhelyi (2012) sought to further utilize data from the 100-Car Study to identify a new measure, critical jerk, for analysis of near-crash events. The need for this type of analysis stems from the regard that the large amount of data collected in the research takes a very long time to traditionally process. The re- 
search was specifically aiming to more accurately identify near-crash events compared to a previous definition of near-crash established by previous researchers that used longitudinal acceleration. The new methodology utilizing critical jerk was proven to perform 1.6 times more accurately compared to the previous longitudinal acceleration method. This research concluded that the measurement of critical jerk was capable of harsh breaking events and could also be utilized for assessing higher risk drivers.

\section{SHRP2}

Currently in progress, the next major naturalistic driving study performed in the United States is the Strategic Highway Research Program 2 (SHRP2). The major goals associated with SHRP2 are to gain increased knowledge of driver behavior through naturalistic driving data. Through the observation of driver behavior, researchers hope to better understand how drivers interact with various driving conditions, identify crash risk associated with driver's interaction with various conditions, and propose appropriate counter measures based on findings. The instrumentation utilized in this research is similar in nature to the 100 -car study, including detailed sensor data and multiple video feeds of drivers. A total of 3,100 participants are included in the study, and a total of 1,950 instrumentation packages. The total study is expected to collect 3,900 vehicle-years of data from 6 study areas across the United States. The total dataset is expected to be over 1 petabyte ( 1 million gigabytes) of data. Aside from the significantly larger amount of data being recorded with SHRP2, this new research will differ from the 100Car Study in that data is collected in multiple geographic areas with multiple 
types of vehicles, including vans, sport utility vehicles, and pick-ups. (Campbell, 2010) While the majority of the data has currently been collected, little in terms of analysis has been published at the time of this thesis.

\section{CONCLUDING REMARKS}

This literature review shows previous work performed in this area and helps to demonstrate some key elements unique to this research. For one, the major naturalistic driving studies performed require extensive vehicle instrumentation, while this study simply requires GPS driving data obtainable from a variety of sources. For the Two-Fluid model analysis, this research differentiates itself from previous work by Hong et al. (2005) in the regard that this study utilized multiple drivers with GPS devices that did not require affixing to the vehicle. With regards to analysis of highway segments, this research differentiates itself from previous work by Guo and colleagues (2010) due to the fact that identification or real-time classification of individual near-crash events is not the goal of utilizing the measurement of vehicle jerk. This research, instead, uses the measurement of jerk to determine which segments see higher percentages of higher jerk events and attempts to correlate this information with long-term traffic safety performance of roadways. 


\section{DATA COLLECTION AND PROCESSING}

The data used for this study was collected from a naturalistic driving study performed in San Luis Obispo. All participants in the study were staff members from Cal Poly. The total data collection period lasted from July 2012 to March 2013. Each participant in the study was given a GPS device for their personal commute vehicle for a period of approximately two weeks. While GPS data recorded continuously as the vehicle was in motion, this study focuses on a few selected roadways within the city limits of San Luis Obispo. The selected roadways include two arterial roadways and an urban highway to demonstrate the applications of this data. This chapter further describes the study roadways of interest, the GPS devices, the attributes of the participants of this study, and the data processing.

\section{STUDY ROADWAYS}

The particular roadways of interest in the research were selected based on the large frequency of trips observed within the complete GPS dataset. The two arterial roadway of interest are California Boulevard and Grand Avenue. These arterial roadways are both multi-lane arterial roadways near Cal Poly. The highway segment of interest is a 5-mile long section of US Highway 101 within the city limits of San Luis Obispo. Figure 1 shows the selected study areas for Grand Avenue and California Boulevard. Figure 2 shows the segment of US Highway 101 of interest. 


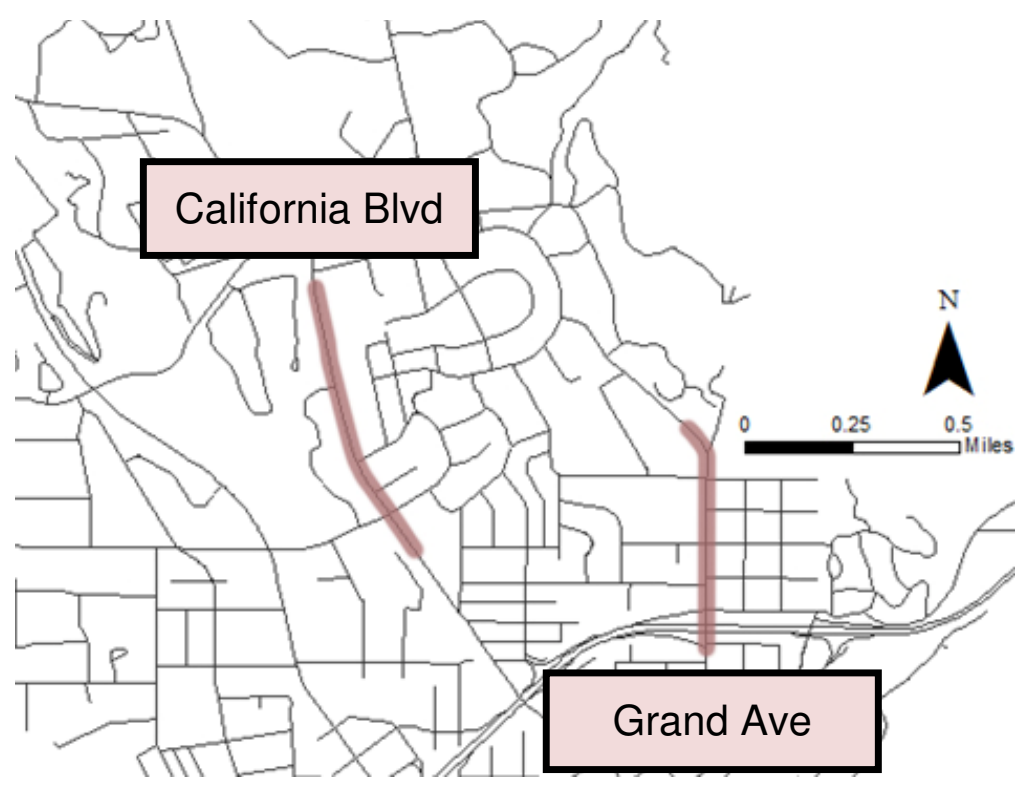

Figure 1. Arterial Segments of Interest: Grand Avenue and California Boulevard

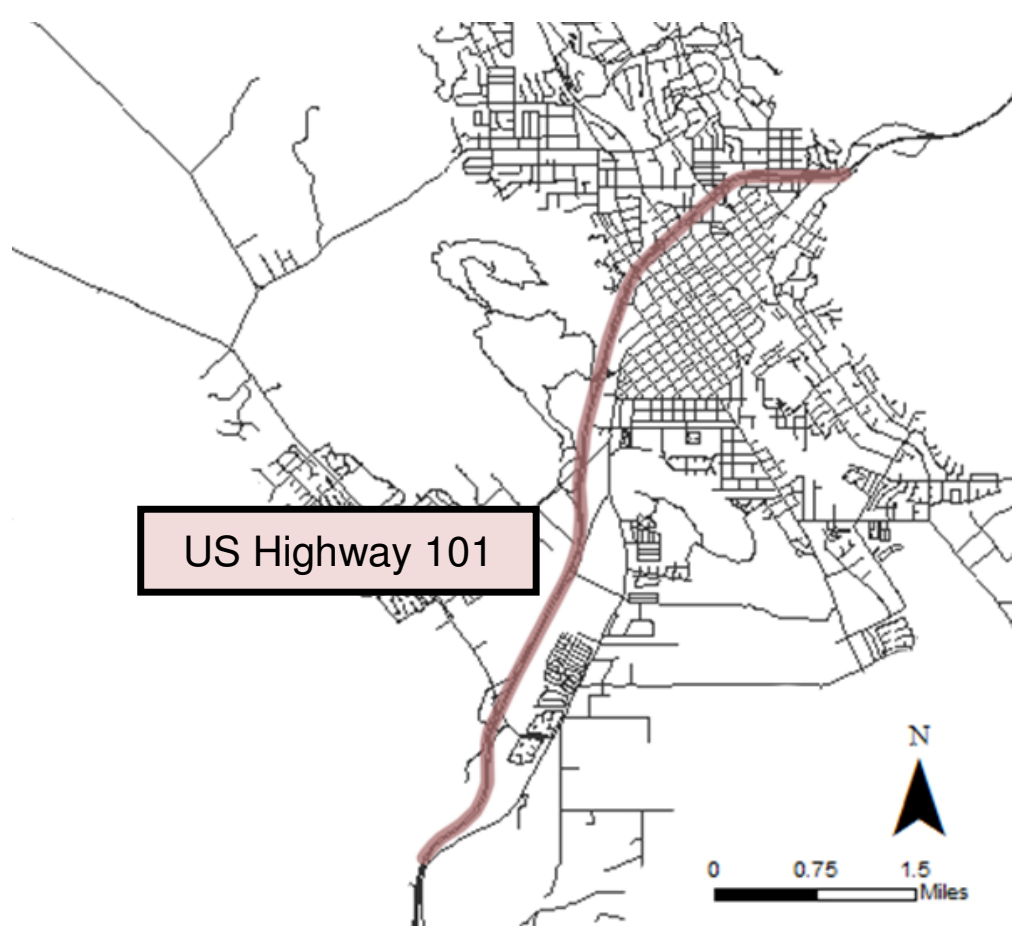

Figure 2. Highway Segment of Interest: US Highway 101 


\section{DATA COLLECTION AND PREPARATION}

\section{Participant Information}

Driving participants in this study were all staff members of Cal Poly. Participants were initially selected using an online screening questionnaire which solicited both personal and driving information. Using information provided on the questionnaire, potential participants were segregated based on specific age groups, typical route selections, and driving frequency. Selected participants were given further information prior to their participation to verify they understood the GPS devices, the information being recorded, and their rights. Participant confidentiality was guaranteed to the extent for which the law permitted.

Desirable participants for this study were individuals who commuted to Cal Poly multiple times per week. A total of 33 participants were enlisted to serve as drivers in this study. These participants ranged in age from 25 to 55 years of age, and included 23 female drivers and 10 male drivers. Participants lived in various cities and communities within the counties of San Luis Obispo and Santa Barbara. Figure 3 below shows a histogram of the various home locations of drivers for which they typically commuted to and from. 


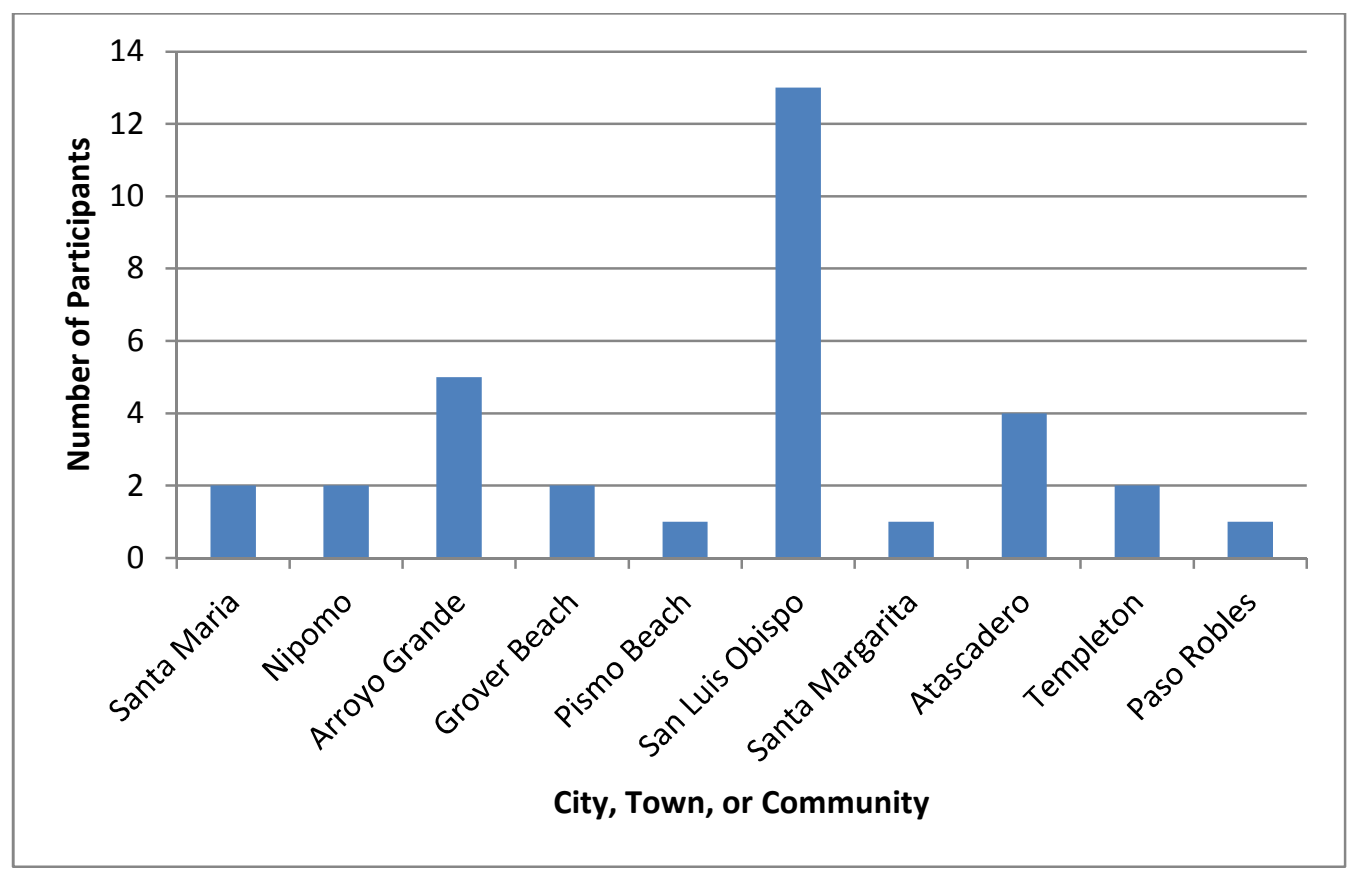

Figure 3. Histogram of Participant Residences

As stated before, each participant kept the GPS device in their vehicle for a period of two complete weeks. Participants were asked to refrain from allowing other individuals to drive their vehicle during the two week period. Participants were given no additional instructions relating to their style of driving or the routes they selected.

\section{GPS Device Information}

The OHARARP SD GPS Data Logger V3.15 (data logger) was selected as the GPS device to collect data in this study. A total of ten GPS data loggers were purchased and employed for data collection. These GPS units were manufactured by OHARARP, LLC and were selected for use in this study because of their ease of implementation, user friendly interface, and sufficient battery life. One GPS data logger is pictured in Figure 4. 


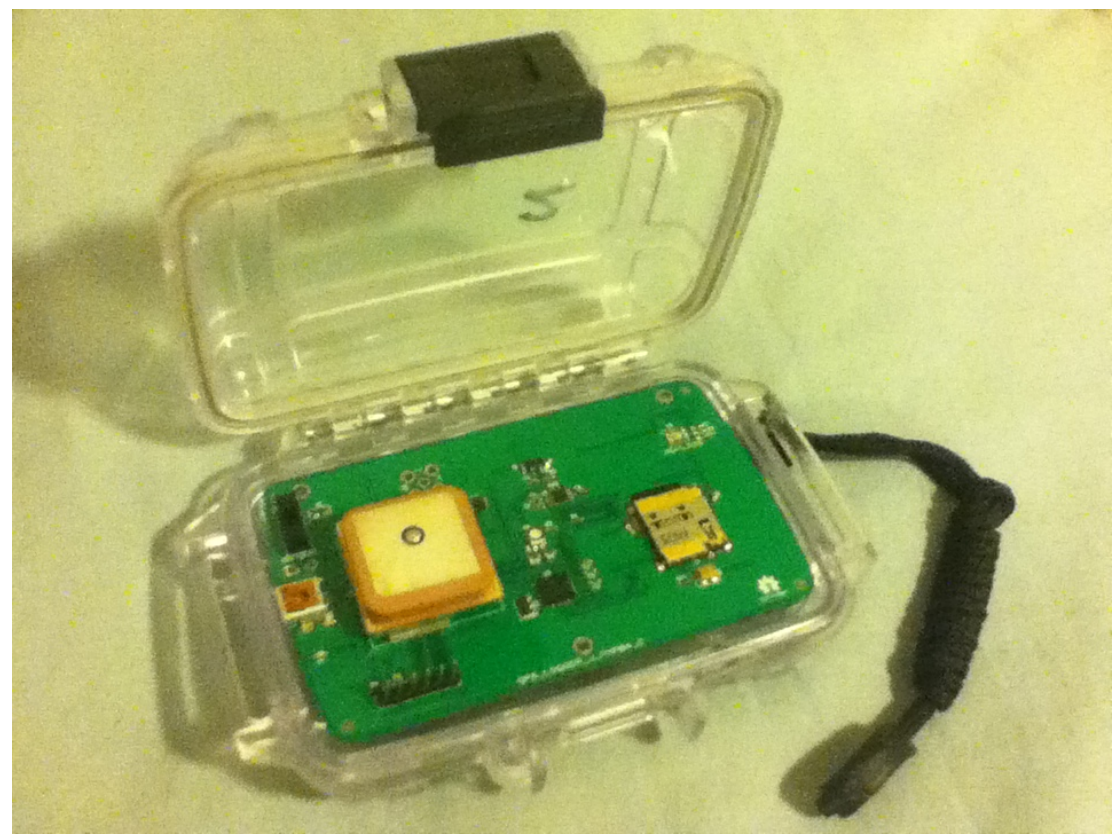

Figure 4. OHARARP SD GPS DataLogger V.315

The data loggers were installed with a FMP04-TLP GPS modulus processing chip manufactured by fTech. The devices were powered by a rechargeable 1800 mAh 3.6V lithium ion battery. An 8 gigabyte micro SD memory card stored the collected data while a multi-color LED light indicated functionality of the data logger. The circuit board and GPS chip were protected in a protective case to assure they were not damaged during the data collection.

GPS loggers were programmed to record in comma-separated value (CSV) file format from parsed sentences that followed National Marine Electronics Association (NMEA) standards GPGGA, GPGSA, GPGSV, and GPRMC. All data was recorded an average rate 2.5 hertz based on the settings of the data loggers. The GPS information recorded by each data logger is provided in Table 3. 


\section{Table 3. GPS Data Logger Attributes Recorded}

\begin{tabular}{|l|c|c|}
\hline \multicolumn{1}{|c|}{ Attribute } & Attribute Description & Remark / Unit \\
\hline LATITUDE & Latitude & WGS84, Degrees $\left(^{\circ}\right)$ \\
\hline LONGITUDE & Longitude & WGS84, Degrees $\left(^{\circ}\right)$ \\
\hline ALTITUDE & Altitude & WGS84, Meters (m) \\
\hline HEADING & Directional Heading of Movement & Degrees $\left(^{\circ}\right)$ \\
\hline SPEED & Velocity of Device & Miles Per Hour (MPH) \\
\hline SAT & Number of Satellites in Communication & \\
\hline PDOP & Positional Dilution of Precision & \\
\hline HDOP & Horizontal Dilution of Precision & \\
\hline VDOP & Vertical Dilution of Precision & \\
\hline FIX & Recording Status & \\
\hline YEAR & Current Year & UTC \\
\hline MONTH & Current Month & UTC \\
\hline DAY & Current Day & UTC \\
\hline HOUR & Current Hour \\
\hline MIN & Current Minute & UTC \\
\hline SEC & Current Second & UTC \\
\hline MSEC & Current Millisecond & UTC \\
\hline
\end{tabular}

Latitude and longitude were recorded by the devices to the standards of the 1984 update of the World Geodetic System (WGS84). The total number of significant figures which latitude and longitude were recorded varied due to a firmware error programed into some of the devices, but never exceeded 10 significant digits. The attribute ALTITUDE recorded to the nearest tenth of a meter. The attributes HEADING (degrees) and SPEED (MPH) were recorded with up to 2 digits after the decimal place. The attribute SAT, representing the total number of satellites in communication with the device, was a single number while the three dilution of precision values (PDOP,HDOP,VDOP) were recorded with 4 significant figures. FIX, representing the recording status of the device, was a binary 0 or 1 attribute depending on whether or not the device was actively communicating with a minimum of 4 GPS satellites. Time values recorded by the device (including YEAR, 
MONTH, DAY, HOUR, MIN, SEC, MSEC) followed Coordinated Universal Time (UTC) and was reported to the nearest one tenth of a second. A sample of data being outputted by the GPS devices is shown in Appendix A of this thesis.

Battery life on the GPS data loggers varied based on participant driving frequency and many other conditions. GPS data loggers were not recharged by researchers or participants during the two-week period. To preserve battery, each data logger was programmed with a sleep mode that disabled data recording if the device remained idle for a period greater than 300 seconds. GPS data loggers that frequently lost communication with available satellites drained battery life faster compared to those with easier access to satellite communication. It was observed that typical battery life of each data logger lasted 9 to 11 complete days.

\section{GPS Data Collection Process and Associated Errors}

During the experimentation process the data loggers needed to be strategically placed in the vehicles to prevent them from moving independently of the vehicle. Data loggers also need to be placed in a location that did not block driver's line of sight or act as a visual distraction. Typically, the GPS loggers were positioned in vehicle center consoles or glove boxes. By initial testing it was determined that placing the logger in either location did not impact GPS communication.

GPS data loggers proved to record data considerably well despite various physical obstructions and weather conditions. Values of HDOP and PDOP remained below a value of 2 for over $85 \%$ of the data from all participants. With this said, 
certain types of GPS recording errors were found in the complete dataset. These errors could generally be categorized in one of three ways: noise, wandering, or gaps. GPS noise, which occurred while the vehicle was stopped or traveling at low speeds, was the most common error observed in the data. This error occurred when changes in GPS positional data (latitude and longitude) and speed data did not accurately reflect the true movement of the vehicle. This error typically resulting in a large "cluster" of data points with inaccurate speed measurements observed around the true location of the vehicle. GPS data wandering was also observed in some datasets. Wandering occurred when the GPS positional data significantly differed from the true location of the vehicle while the vehicle was traveling at higher rates of speed. Wandering typically was a random error and was identified by observing vehicle seemingly traveling on roadways that did not physically exist. Large gaps in the GPS data also were seen; these errors were caused by a sudden lack of GPS satellite availability due to some impedance or communication malfunction. Figure 5 shows these various error types. 


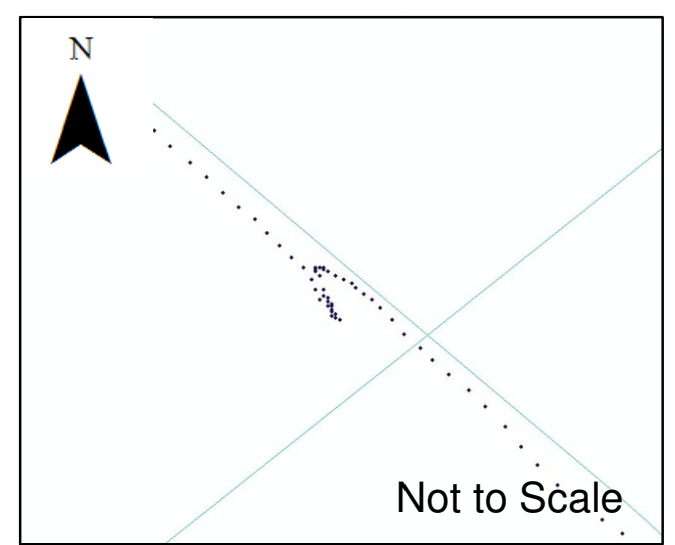

a) GPS Noise at Intersection

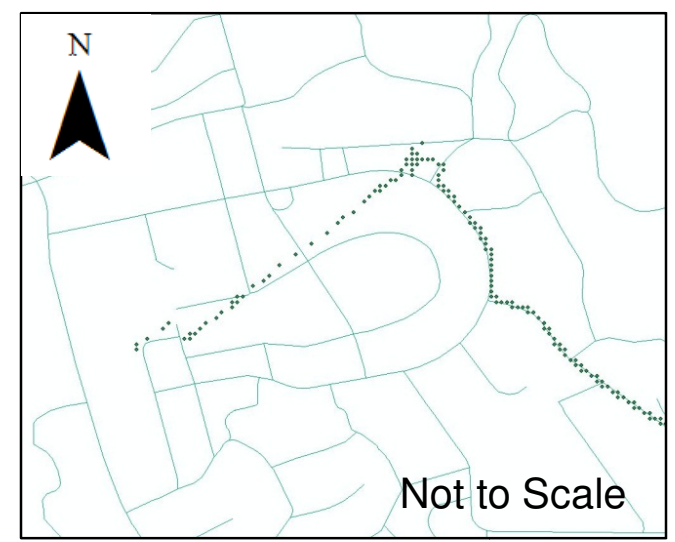

b) GPS Wandering

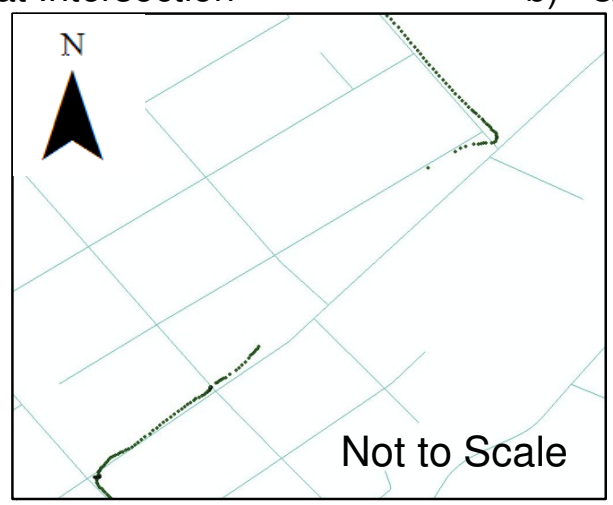

c) GPS Gap

\section{Figure 5. Types of GPS Errors}

GPS driving data collected by the data loggers was not accompanied with any other driving data sources. This includes no live video feed, no additional motion sensors (such as accelerometers), or information relating to traffic conditions. Data relating to the make and or model of the specific vehicles used by participants was not recorded.

\section{Data Processing and GIS Linear Referencing}

GPS data collected from each study participant was processed by combining multiple data files from the data logger into a single file named according to a 
random identification code. Driver attributes (including gender, age, commuting behavior, etc.) collected in the initial questionnaire were linked with GPS data. Each GPS observation in the database was assigned a "trip number" based on the relative time difference between successive GPS data point readings for the purpose of separating individual trips. A new trip number was assigned to an observation if the time difference from the last data point was greater than 30 minutes. Individual participants' GPS data was imported into ESRI ArcMap and overlaid onto a San Luis Obispo County GIS roadway base map. This GIS base map was assumed to represent the true locations of various roadway locations and geometric features. Figure 6 shows the GIS base map utilized for the remainder of the data processing.

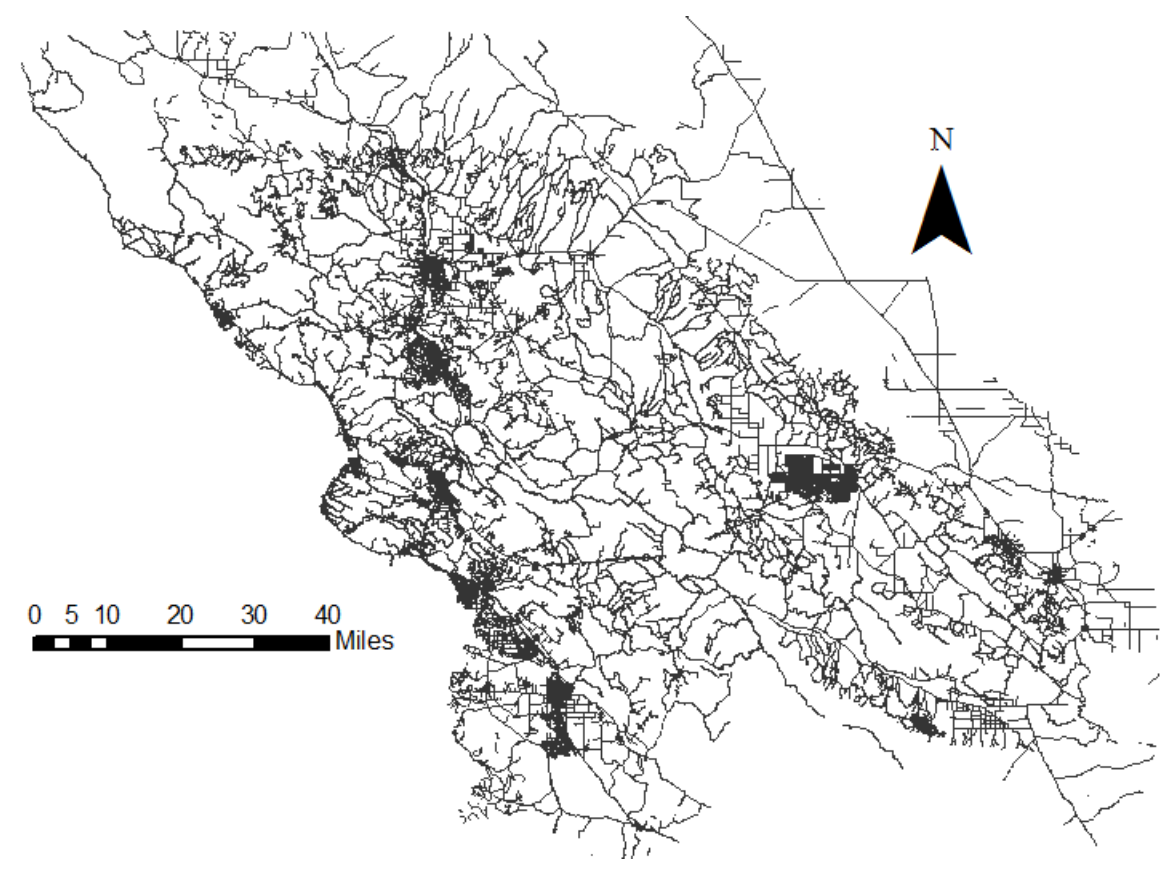

Figure 6. San Luis Obispo County GIS Roadway Base Map 
Despite the precision of GPS latitude and longitude values which the data loggers were capable of recording, GPS data points often did not lie on roadway locations shown on the GIS base map or on GIS based photogrammetry. The amount of variation from a true roadway location to recoded location varied significantly based on GPS satellite coverage, GPS impedance, speed of the device, and many other factors. This lack of accuracy in the data precluded the ability to detect lane changing events or which lane a participant was in during a given reading.

Because of these spatial anomalies in the data, a consistent methodology needed to be implemented to ensure proper GPS data was being utilized for analysis relating to individual roadways. The process of linear referencing available in ArcMAP was selected as the methodology to approach this problem. Linear referencing is a spatial analytical technique for storing and referencing point events relative to their position along a measured route (McCracken and Law, 2008). The process of linear referencing creates a linear axis for which GPS data points could be spatially compared with one another. Linear referencing also allows for multiple data sources to be processed, meaning that other data sources (such as crash data) could be linearly referenced to a common axis with GPS data.

Using the road network base map, individual roadway segments were merged together to form individual polylines for each roadway feature of interest. Linear reference routes were created from the merged polyline features by using the "create routes" tool in ArcMap. A total of 6 individual routes were created, two routes for Grand Avenue, two routes for California Boulevard, and two routes for 
US Highway 101. Note that two individual routes needed to be created for each roadway of interest to obtain different spatial locations for each direction of travel.

After processing GIS routes, the "locate features along route" tool in Arcmap assigned each GPS data point with the spatial information from a selected route. Each GPS data point was linear referenced based on a shortest distance equation, meaning that the data point was assigned the spatial attribute of the point along the route for which was the closest distance to that route. A data "search radius" of 300 feet was implemented for the purpose of linear referencing, meaning that all data within 300 feet of the selected route was linear referenced to that route. After linear referencing was complete, another GIS process called dynamic segmentation was utilized to "shift" GPS data points from their original latitude and longitude values to new latitude and longitude values that corresponded to the point they were linear referenced to. Figure 7 shows an illustration of the GPS data before and after the implementation of linear referencing and dynamic segmentation. 

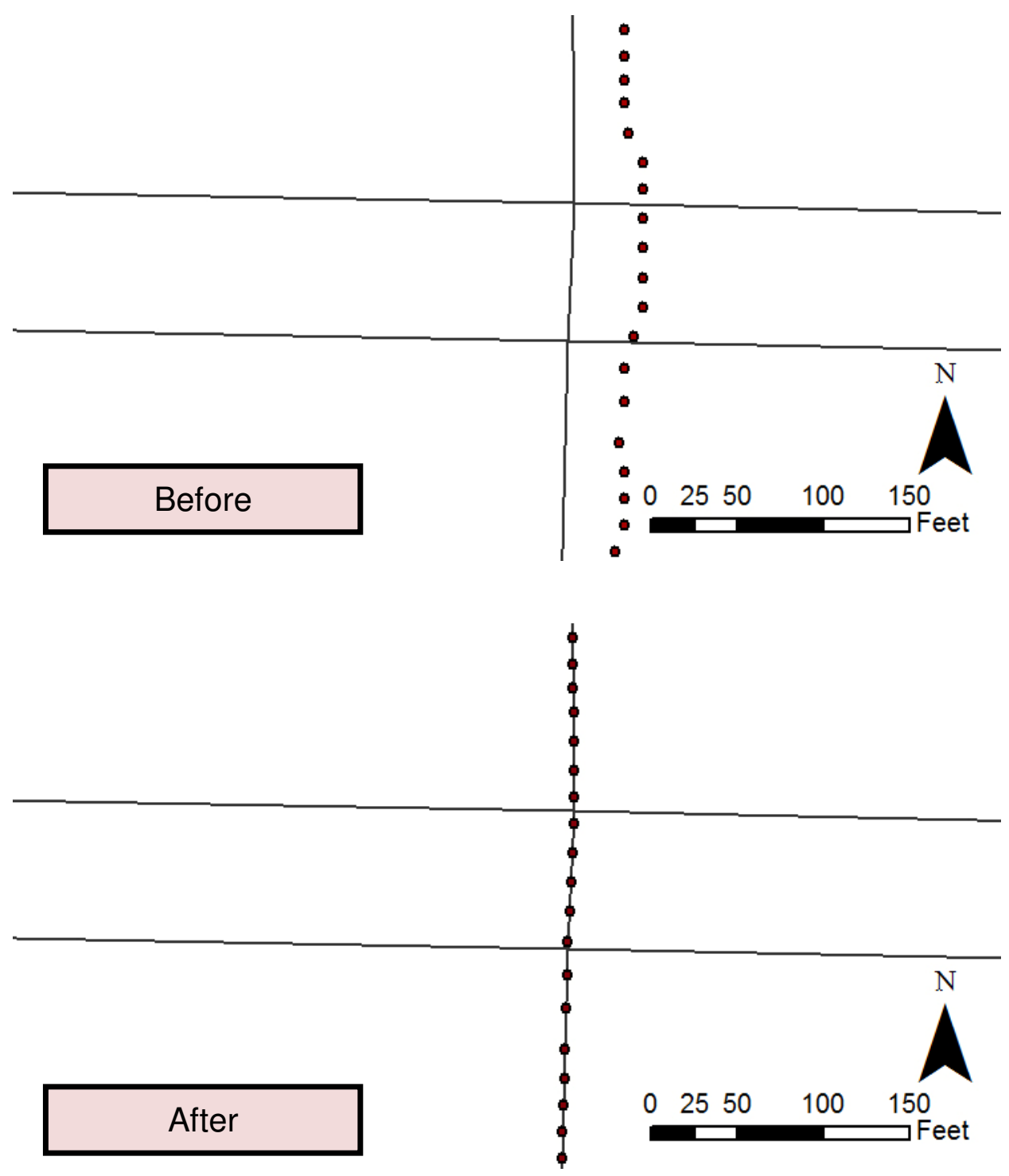

Figure 7. Linear Referencing and Dynamic Segmentation Example

The linear referencing and dynamic segmentation produced multiple GPS database tables with the new spatial attribute along each route. Because actions such as lane changing could not be determined (due to the lack of necessary accuracy), the total distance traveled by participants between data points was taken as simply the difference in linear referenced distances. This assumption means 
that all data recorded by the device is longitudinal in nature, meaning that there was no travel performed by the participant follows the GIS base map route without any variation. This assumption not only applies not only to positional attributes along the route, but also for vehicle speed.

Additional logical rules were applied to the output tables to further verify the quality of the data for analysis purposes. First, filtering any data with HDOP values greater than 3 ensured that GPS data collected during unreliable periods of satellite communication was not used in further analysis. Secondly, logic rules were implemented to remove any data points with incorrect heading (direction of movement) attribute.

\section{CONCLUDING REMARKS}

This chapter described the study areas, GPS devices, data collection, and data processing efforts made. As with many research efforts, the success of data collection and processing is vital to the success of the analysis. The methodology and study design described here reflects careful consideration of the dataset and the objectives of the research. 


\section{TWO-FLUID MODEL ANALYSIS}

The first section of analysis for this study was desired to be conducted for arterial roadways. Based on previous research, the Two-Fluid model was selected as the model of choice for arterial roadway analysis. This decision to utilize the TwoFluid model comes from previous research that has shown correlation between the Two-Fluid model and traffic accidents of varying severities (Dixit et al., 2011). The Two-Fluid model parameters were determined for the two arterial roadways of interest, Grand Avenue and California Boulevard, by utilizing linearly referenced GPS data to determine total stop time and travel time for individual trips along these routes. The unit length of segments for this research was 0.5 miles $(0.8 \mathrm{~km})$ each.

As discussed in Chapter 2, previous research by Hong et al. (2005) proved the applicability of GPS devices to obtain Two-Fluid parameters. While this research is utilizing GPS data for Two-Fluid model parameters, it differs from this research in a few regards. First, previous research was proven for a street network and not individual arterial roadways as in this research, and also Hong et al.'s research did not have a large sample size of participants as this research has done. Previous research by Hong et al. failed to employ the same data processing methods as described in Chapter 3, such as linear referencing.

\section{TWO-FLUID MODEL}

Prigogine \& Herman (1979) developed the Two-Fluid model after years of observing vehicular traffic on multi-lane roadway facilities. Their model was a macroscopic flow model that viewed traffic flow in a network as a collection of 
stopped and running vehicles. The Two-Fluid model works under two general assumptions about vehicle traffic. The first assumption is that the average running speed in a street network is proportional to the fraction of vehicles that are moving. The second assumption is that the fractional stop time of a probe vehicle circulating in a network is equal to the average fraction of the vehicles stopped during the same period.

As described earlier in Chapter 2, the major outputs of the Two-Fluid model are the parameter known as $n$ and the parameter known as $T_{m}$. The formal Two-Fluid model formulation is provided in Equation 1, and is transformed into the natural logarithmic equivalent in Equations 2 and 3 (Herman and Ardekani, 1984).

$$
\begin{aligned}
& T_{S}=T-T_{m}^{\frac{1}{n+1}} * T^{\frac{n}{n+1}} \\
& T_{R}=T_{m}^{\frac{1}{n+1}} * T^{\frac{n}{n+1}} \\
& \ln T_{R}=\frac{n}{n+1} \ln T+\frac{1}{n+1} \ln T_{m}
\end{aligned}
$$

where:

$T_{s}: \quad$ Stop time

T: $\quad$ Average travel time

$T_{R}: \quad$ Running time $\left(T-T_{s}\right)$

$T_{m}: \quad$ Average minimum trip time per unit distance

$n: \quad$ Indicator of the quality of traffic service in network

It is through the natural logarithmic equivalent that the Two-Fluid parameters can 
be obtained. It can be observed that Equation 3 can be re-written as Equation 4, and that the Two-Fluid parameter can be obtained from Equation 5 and Equation 6 (Herman and Ardekani, 1984).

$$
\begin{aligned}
& \ln \left(T_{R}\right)=B * \ln (T)+A \\
& n=\frac{B}{1-B} \\
& T_{m}=e^{\frac{A}{1-B}}
\end{aligned}
$$

As discussed in Chapter 2, the Two-Fluid parameters of $n$ and $T_{m}$ have been shown to correlate with total crash rate (Dixit et al., 2011). By obtaining the TwoFluid parameters, a few deductions about the long-term traffic safety of these two arterial roadways can be made. For this analysis, it is assumed that the GPS driving data collected represents average driving that is not conservative or aggressive in nature.

\section{DETERMINATION OF TWO-FLUID PARAMETERS}

Linearly referenced GPS data sets were processed in Microsoft Excel to obtain Two-Fluid parameters. An Excel spreadsheet used a set GPS data point to act as the beginning of the $1 / 2$-mile trip, and calculated the total time difference between the beginning point and a point that was over $1 / 2$-miles in total length. The spreadsheet identified any data that had speed attributes less than a certain threshold, and deemed these data points as "stop" activity. 
A threshold value of $2 \mathrm{MPH}$ was utilized for this analysis. The need to determine a threshold value for stop comes from GPS noise that occured with GPS data collected at low speeds. The determination to use $2 \mathrm{MPH}$ as the threshold value of choice came from observations in initial testing, and was deemed to properly capture light braking events. This threshold of $2 \mathrm{MPH}$ was in the range of the threshold of $2 \mathrm{KMPH}$ utilized by Hong et al. (2005).

Using the stopping threshold, total travel time and stopped time for each trip was obtained. Graphical representation of the data points is shown in Figure 8 and Figure 9. The Two-Fluid model parameters for the two arterial roadways are summarized in Table 4.

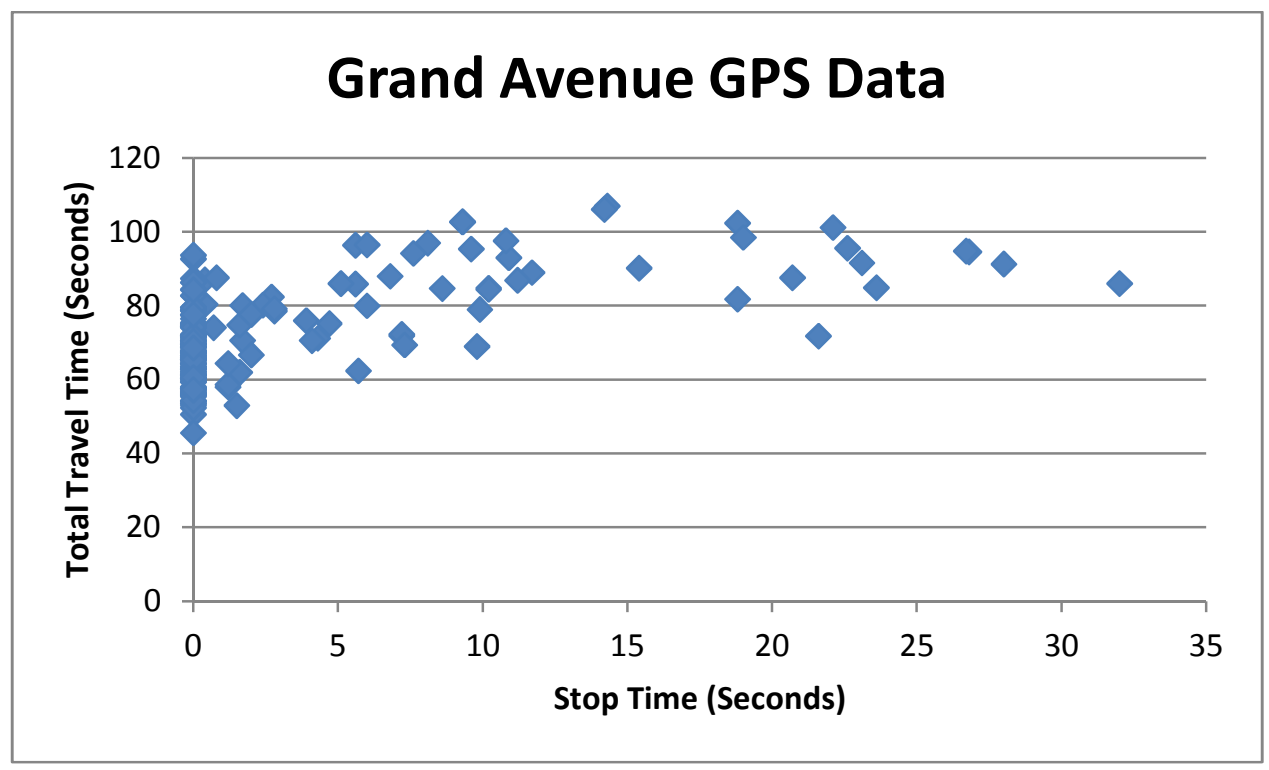

Figure 8. Relation between Total Travel Time and Stop Time for $1 / 2-$ Mile Trips on Grand Avenue 


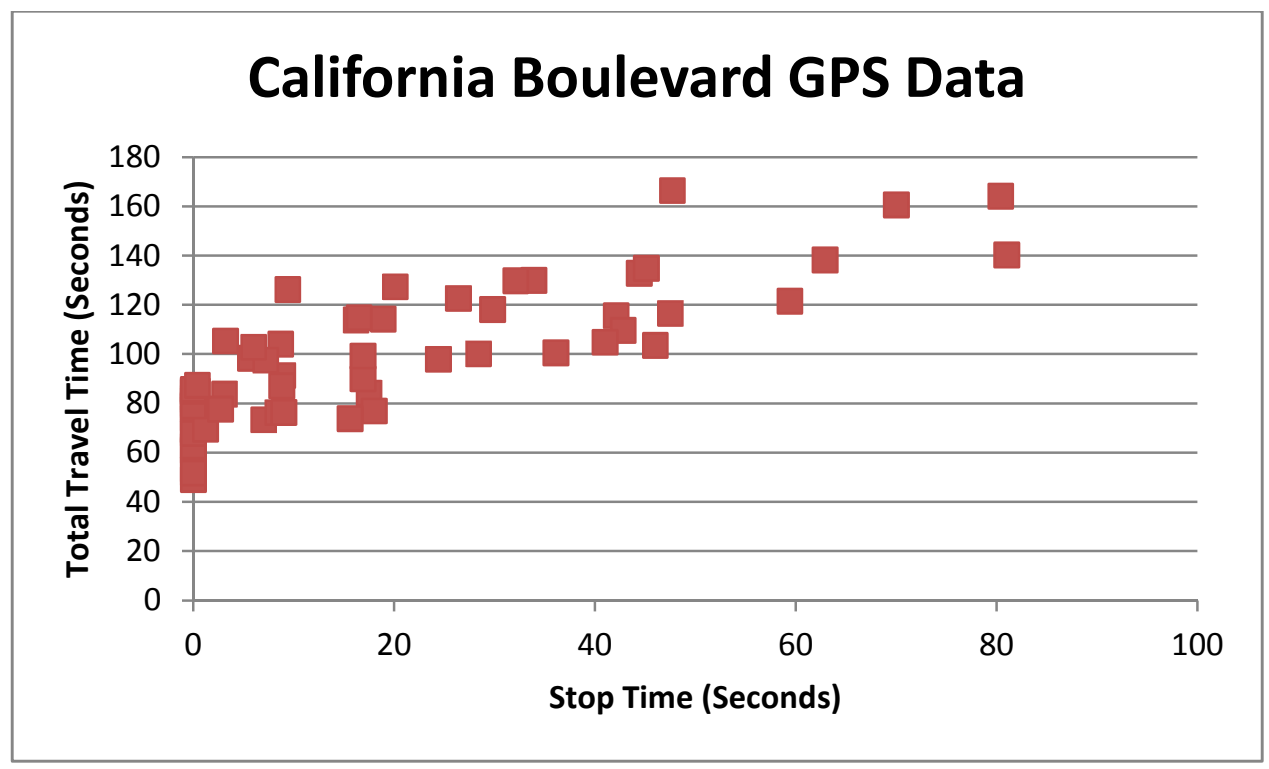

Figure 9. Relation between Total Travel Time and Stop Time for $1 / 2-$ Mile Trips on California Boulevard

Table 4. Two-Fluid Parameters Obtained

\begin{tabular}{|l|c|c|c|c|}
\hline & $\begin{array}{c}\text { Total Number of } \\
\text { Samples }\end{array}$ & $\begin{array}{c}n \\
\text { (Equation 5) }\end{array}$ & $\begin{array}{c}T_{m} \text { (minutes) } \\
\text { (Equation 6) }\end{array}$ & $R^{2}$ \\
\hline Grand Avenue & 179 & 2.81 & 1.02 & 0.38 \\
\hline California Boulevard & 65 & 0.794 & 1.11 & 0.68 \\
\hline
\end{tabular}

As seen from both Figure 8 and 9, the positive curvilinear relationship between stop time and travel time can be seen in the data. Grand Avenue had a larger amount of data points, however the range of observed stopped times for Grand Avenue was much smaller compared to California Boulevard. While the $R^{2}$ values obtained from the GPS data sets were lower compared to some of the other published research, this data was subject to more variability due to multiple drivers' contributions to the data set instead of just one research team with the traditional car chase methodology. It is also important to note that lower $R^{2}$ values 
have been reported by previous researchers as well (Herman and Ardekani, 1984). The sample size of data points collected for Grand Avenue and California Boulevard were deemed to be sufficient as the total sample size was greater than some other published results.

It can be seen from the two figures that a very large percentage of the total data for each arterial was located on the $y$-axis, which occurred when $T_{s}$ was equal to zero. This is unexpected due to the fact that both arterial roadways have at least one stop sign in the study area. While some of these data points may be due to drivers cruising past the stop sign, it is highly unlikely that the entire cluster was caused by this action. The explanation behind the large clusters observed, at least partially, was caused by the GPS noise observed at zero or near zero travel speeds discussed in Chapter 3. Although the $2 \mathrm{MPH}$ threshold for stopping was adapted to accommodate for this noise, it is believed the threshold was not high enough given the nature of the GPS data. Altering the threshold to a higher value would shift more of those data points away from the y-axis, which in turn would alter the obtained $n$ and $T_{m}$ values.

The two values of $n$ obtained from each arterial roadway varied from each other. The $n$ value for Grand Avenue was much higher, which suggests based on previous research that long-term crash rate along Grand Avenue would be higher compared to California Boulevard. This correlation was also observed from the parameter $T_{m}$ as well, which was observed to have a negative correlation with long-term traffic crash rate (Dixit et al., 2011). Therefore, Grand Avenue with a lower value of $T_{m}$ may have a higher crash rate compared to California Boule- 
vard. Verification of these long-term traffic safety trends as proposed by Dixit et al. (2011) could not be proved with this research due to incomplete traffic accident history available from UC Berkeley's Transportation Injury Mapping System (TIMS) database.

\section{DATA VALIDATION}

Two-Fluid parameters obtained from GPS driving data in the California Boulevard study area was validated with data collected in the field utilizing car following techniques described in many other research studies (Herman and Ardekani, 1984). The purpose of data validation was to verify that results obtained from GPS data were not significantly different than those collected using a traditional car chase methodology. This was of particular interest based on the large amount of GPS data points observed with low values of stop time.

In field data collection occurred during the AM and PM peak periods for two separate days along California Boulevard. Figure 10 shows the data points of total travel time versus stopped travel time for data collected utilizing GPS devices and the standard car chase methodology. 


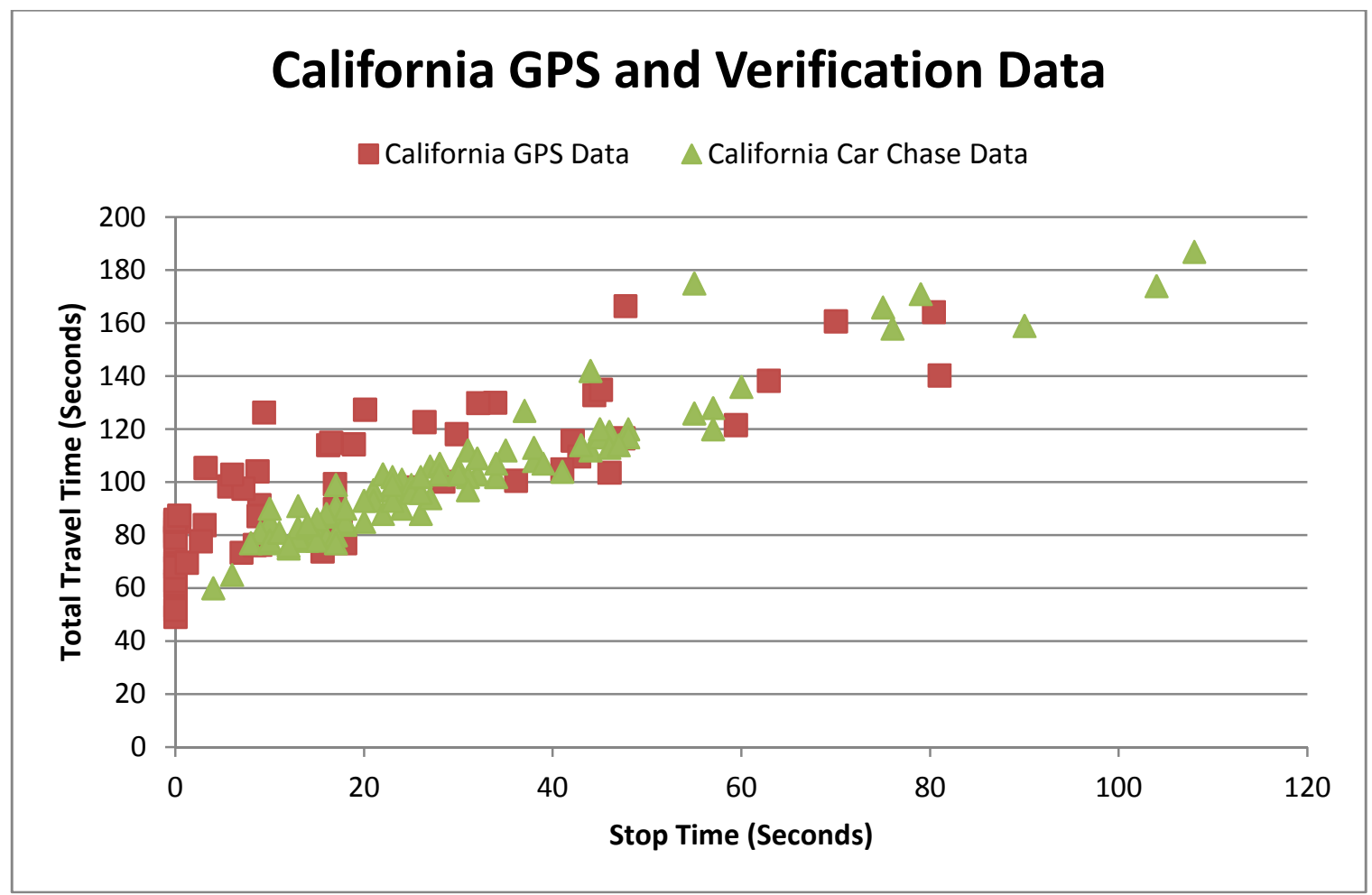

Figure 10. Relation between Total Travel Time and Stop Time for $1 / 2$-Mile Trips on California Boulevard with GPS Device Data and Car Chase Data

As seen in Figure 10, the driving data collected by the car chase methodology resulted in no data points that had any data with stop time equal to zero. This result shown in the validation data was inherently different than what was observed with the GPS data, even with the definition of stop (at a threshold of $2 \mathrm{MPH}$ ) used for the GPS data. Despite this, it can be observed that data collected with higher stop times (i.e. anything greater than 5 seconds of stop time) from either data source tended to lie in the same general area.

A general linear model was performed in SAS to validate the GPS. The test utilized a combined dataset that contained both GPS and validation data and determined if an assigned "verify" variable (which was assigned 1 for verification 
data, 0 for GPS data collected) was significant in the dataset. For this verification, only data with high stop time (when $T_{s}$ was greater than 20 seconds) was considered. If the result of the GLM produced a p-value which was not significant at a $90 \%$ or $95 \%$ confidence level, then the verification attribute can be stated insignificant, meaning there was not statistical difference between GPS data and car chase validation data. Appendix B in this thesis presents the SAS code which was utilized to import the data points after they were transformed, and the code utilized to perform the verification procedure. Table 5 below shows the results of the GLM procedure.

Table 5. Linear Model Data Verification Results

\begin{tabular}{|l|c|c|c|}
\hline Source & DF & F Value & Pr $>$ F \\
\hline Natural Log of TT & 1 & 28.00 & $<.0001$ \\
\hline Verify Data & 1 & 072 & 0.3991 \\
\hline
\end{tabular}

As seen above, the p-value for the "Verify Data" attribute (highlighted in blue) was greater than 0.1 , which suggests that there is no statistical significance between the two data sources. Though with this said, if one was to use the verification data to compute the value of $n$ and $T_{m}$ separate from the GPS data, it is obvious that the car chase data would produce different results. While this fact remains true, the difference between the two data sources was not statistically significant and based on this it was concluded that the GPS data can in fact be used for Two-Fluid model parameter estimation for arterial roadways. 


\section{CONCLUDING REMARKS}

The research conducted for this section of analysis helped to show that GPS data can be utilized for collecting Two-Fluid parameters. With these parameters obtained attributes relating to long term traffic safety can be estimated. While there still needs to be more consideration as to how "stop" is defended, the results of this research are promising. The ability to use GPS driving data can be a much more efficient and easier methodology to collect Two-Fluid parameters, and hence infer about the long-term safety performance of roadways, than the traditional car chase methodology. 


\section{HIGHWAY JERK ANALYSIS}

Highway and freeway facilities are inherently different from arterial roadways, as they are designed to accommodate uninterrupted travel flow. Therefore the TwoFluid model would not be an appropriate model for highway or freeway segments. Previous research (Bagdadi and Varhelyi, 2012) has used the derived measurement of jerk (j), or rate of change of acceleration, to identify harsh braking events (or crash surrogates) within sets of naturalistic driving data. After evaluating this previous research, the derived measurement of vehicle jerk was selected to be the parameter of interest for analysis of highway segments.

It should be noted that the previous research used the measurement of jerk to determine near-crash events with the purpose of crash avoidance in real time. The methodology adopted in this research seeks to examine jerk as a way to determine if a significant correlation can be seen from repeated instances of high vehicle jerk in highway segments and historical crash data from the same segment. The specific methodology was to use the percentage of high instances of jerk above a set threshold, and determine if the locations with higher percentages of high jerk had a correlation with locations of historical traffic crashes.

The area of interest for this portion of analysis is the US Highway 101 within the city limits of San Luis Obispo. The total length of interest was approximately 5 miles long. Analysis for this procedure was conducted by first dividing the 5 miles into several $1 / 4$-mile long segments and then several $1 / 2$-mile long segments. A total of 39 segments were considered for the $1 / 4$-mile analysis, and 19 segments were considered for the $1 / 2$-mile analysis. It was the desire to repeat this analysis 
with these different segment lengths to verify that the results obtained were consistent regardless of segment definition. These segments varied based on multiple factors including geometric features and average daily traffic (ADT).

\section{PROCEDURE}

The procedure for this analysis required two major pieces of information. First historical crash data needed to be collected and processed to give an indication of crash rates within the study segments. Crash data utilized for this analysis was collected from the TIMS database. Historical crash data from the January 2002 to December 2011 where the primary or secondary road was US Highway 101 was used as an indication of long-term safety performance. A total of 297 crashes were observed for the entire analysis area in the ten year time period. Crash counts were converted into a crash rate measure based on observed 2011 ADT values obtained from Caltrans. The 'crash rate' measurement utilized here differs from a traditional crash rate, which normalizes crash frequency with specific annual values of daily traffic. However, due to historical small growth rates of traffic within city limits of San Luis Obispo, it was assumed the measure of 'crash rate' utilized for further analysis was sufficient for this preliminary exploration.

The second set of information needed for this analysis was the data on vehicle jerk obtained from the GPS data loggers. Vehicle jerk was not an output meas-

urement from the GPS device and the values of jerk needed to be calculated from observed GPS readings. The longitudinal acceleration and jerk values were calculated between successive GPS readings using Equations 7 and 8. 


$$
\begin{aligned}
& a=\frac{\Delta v}{\Delta t} \\
& j=\frac{\Delta a}{\Delta t}
\end{aligned}
$$

where:

a: $\quad$ Acceleration $\left(\mathrm{ft} . / \mathrm{s}^{2}\right)$

$\Delta v: \quad$ Change in velocity $(\mathrm{ft} . / \mathrm{s})$

$\Delta t \quad$ Change in time (s)

j: $\quad$ Jerk $\left(\mathrm{ft} . / \mathrm{s}^{3}\right)$

$\Delta a: \quad$ Change in acceleration $\left(\mathrm{ft} . / \mathrm{s}^{2}\right)$

Before analysis with crash rate could be conducted, a value for the jerk threshold needed to be determined. For this study, a low threshold value for vehicle jerk was desired. Using a lower threshold value, in theory, would not only capture data during accident avoidance events, but also driving events where participants had to apply their brakes forcefully yet in a controlled manner. Of course, a lower jerk thresholds values results in higher percentages of the total data that fell above the threshold compared to a higher jerk threshold. For this analysis, only negative jerk events that were observed with deceleration (i.e. $a<0 \mathrm{ft} . / \mathrm{sec}^{2}$ ) events were considered.

Pearson's correlation coefficients were first estimated between calculated crash rate and percentage of high jerk data points for varying jerk thresholds. The code used for this analysis, in addition to the other modeling techniques for the high- 
way analysis, is provided in Appendix C of this thesis. The Pearson's coefficients were established to assess if a positive correlation between the two measurements existed. The Pearson's coefficients were also utilized to determine which jerk threshold produced the highest correlation with calculated crash rate for both the $1 / 4$-mile and $1 / 2$-mile long segments. Figure 11 shows the calculated Pearson's correlation coefficients for jerk thresholds varying to $-4 \mathrm{ft} . / \mathrm{s}^{3}$ in increments of .5 $\mathrm{ft} . / \mathrm{s}^{3}$ for both the $1 / 4$-mile and $1 / 2$-mile long segments.

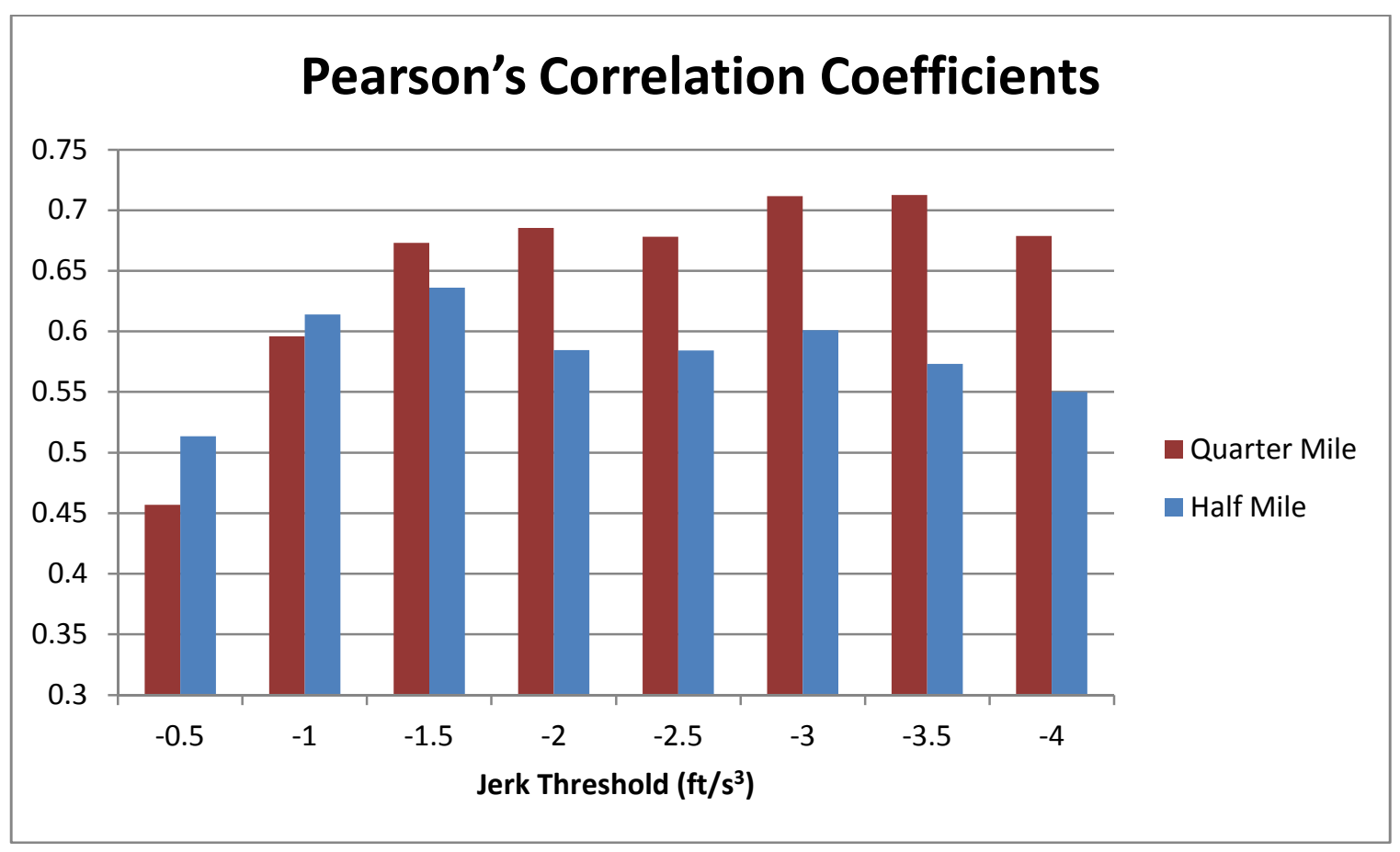

Figure 11. Pearson's Correlation Coefficient for varying jerk thresholds

Figure 11 above shows that the Pearson's correlation coefficient tends to increase and then level out past a certain threshold value. As expected, all of the correlation coefficients are positive and were statistically significant. For the $1 / 4^{-}$ mile analysis it appears that the correlation coefficient leveled out at a jerk 
threshold of $-2 \mathrm{ft} . / \mathrm{s}^{3}$. The $1 / 2-$ mile segment analysis this leveling occurred at the threshold of $-1 \mathrm{ft} . / \mathrm{s}^{3}$. The values where this leveling occurred were set to be the jerk threshold for further analysis.

Using the jerk threshold, negative binomial regression models were employed to determine the significance of the high jerk percentage measure in estimating total observed crashes. Negative binominal modeling is a well-established method for crash frequency estimation that has been seen in previous studies (Milton and Mannering, 1998; Abdel-Aty and Radwan, 2000). The jerk value for which leveling was observed in the Pearson's correlation coefficients was taken to be the jerk threshold for the negative binomial regression models. This meant that for the $1 / 4$-mile analysis, the percentage of observations with vehicle jerk less than -2 $\mathrm{ft} . / \mathrm{s}^{3}$ and negative acceleration was calculated and used as the explanatory variable in the crash frequency analysis. For the $1 / 2$-mile analysis, the percentage of observations with vehicle jerk less than $-1 \mathrm{ft} . / \mathrm{s}^{3}$ and negative acceleration was calculated and used as the explanatory variable in the crash frequency analysis.

\section{QUARTER MILE ANALYSIS}

Analysis for the $1 / 4$-mile segment data included 20 segments in the NB direction of US Highway 101 and 19 segments in the SB direction. The 1/4-mile analysis segments defined in this study are shown in Figure 12 below. 

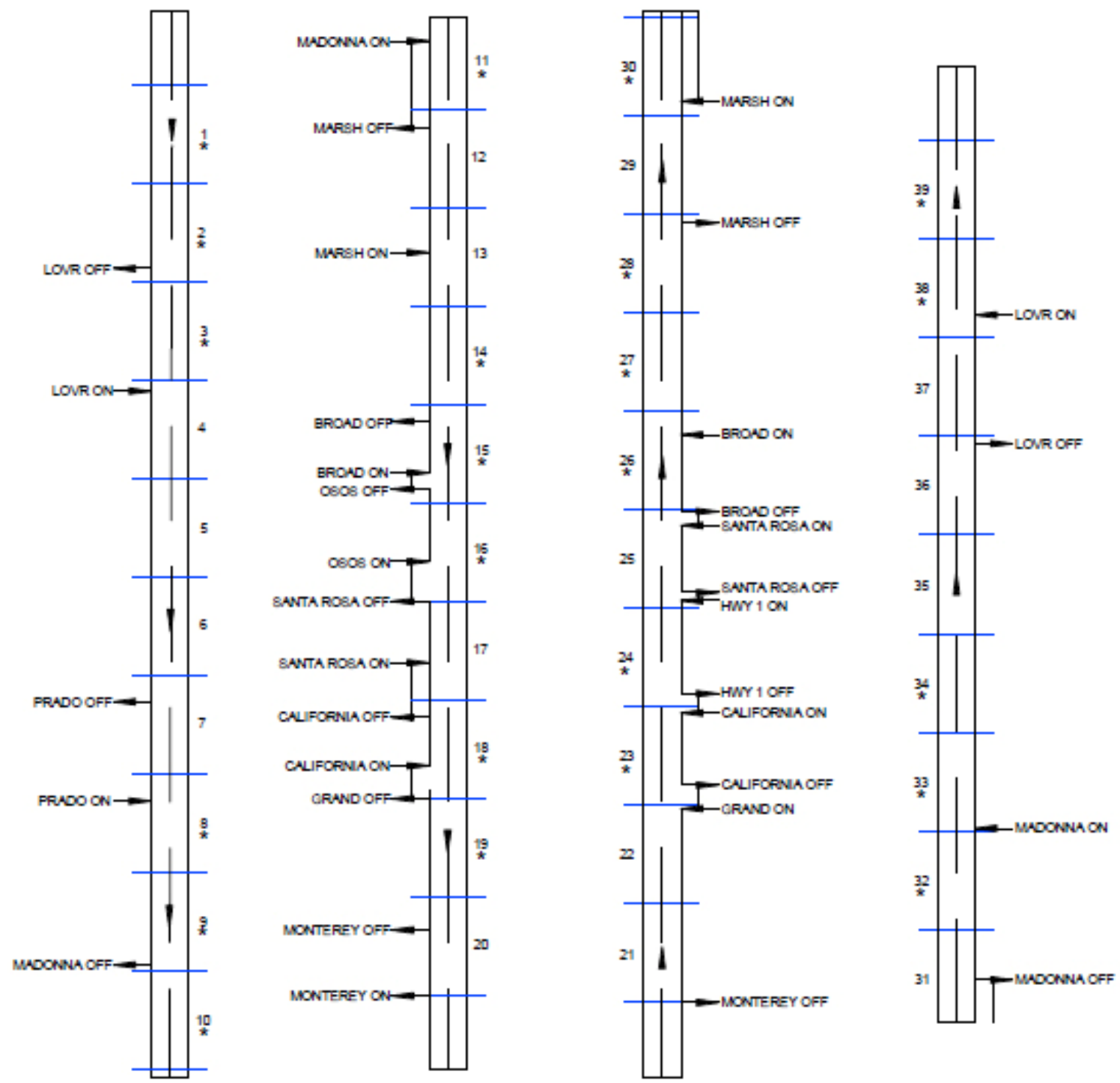

Figure 12. Straight line diagrams for US NB (left, 1-20) and SB (right, 21-39) quarter-mile segments

A total of four negative binomial regression models were estimated for the $1 / 4$-mile analysis segments. The first model used the high jerk percentage as its only variable for estimating total number of crashes. The second model used three other factors relating to each individual segment, presence of curve, presence of weaving (as indicated by an auxiliary lane), and ADT, for total crash estimation. The 
third model utilized the percentage of high jerk events in addition to the other three factors used in Model 2. The fourth model used ADT and high jerk percentage as two explanatory variables. For these negative binomial regression models, the dependent variable of analysis was total crash count. Crash count was used as the dependent variable for negative binomial model analysis to allow the addition of ADT to be included as a possible independent variable. Table 6 below highlights the results of the negative binomial models with significant $p$-value at a $95 \%$ confidence level highlighted. 


\section{Table 6. Crash Estimation utilizing Negative Binomial Regression Models for Quarter Mile Segments}

\begin{tabular}{|c|c|c|c|c|}
\hline \multicolumn{5}{|c|}{$\begin{array}{l}\text { Quarter Mile Segment Model } 1 \\
\text { Negative Binomial Regression Model with High Jerk Criteria } \\
\text { AIC (Akaike Information Criterion): } 230.82 \\
\text { Analysis of Maximum Likelihood Parameter Estimates }\end{array}$} \\
\hline Parameter & Estimate & $\begin{array}{l}\text { Standard } \\
\text { Error }\end{array}$ & $\begin{array}{l}\text { Wald Chi } \\
\text { Square }\end{array}$ & $\mathrm{p}$-value \\
\hline Intercept & 1.3580 & 0.2518 & 29.09 & $<.0001$ \\
\hline High Jerk Percentage & 0.1297 & 0.0449 & 8.36 & 0.0038 \\
\hline Dispersion & 0.2894 & 0.0998 & & \\
\hline \multicolumn{5}{|c|}{$\begin{array}{l}\text { Quarter Mile Segment Model } 2 \\
\text { Negative Binomial Regression Model with ADT and Geometric Variables } \\
\text { AIC (Akaike Information Criterion): } 242.57 \\
\text { Analysis of Maximum Likelihood Parameter Estimates }\end{array}$} \\
\hline Parameter & Estimate & $\begin{array}{l}\text { Standard } \\
\text { Error }\end{array}$ & $\begin{array}{l}\text { Wald Chi } \\
\text { Square }\end{array}$ & $p$-value \\
\hline Intercept & 1.504 & 0.8021 & 3.52 & 0.0608 \\
\hline Curve & 0.0183 & 0.2532 & 0.01 & 0.9418 \\
\hline Weaving & 0.3008 & 0.3236 & 0.86 & 0.3526 \\
\hline Average Daily Traffic & 1.6972 & 2.7316 & 0.39 & 0.5344 \\
\hline Dispersion & 0.3971 & 0.1185 & & \\
\hline \multicolumn{5}{|c|}{$\begin{array}{l}\text { Quarter Mile Segment Model } 3 \\
\text { Negative Binomial Regression Model with High Jerk, ADT and Geometric Variables } \\
\text { AIC (Akaike Information Criterion): } 234.46 \\
\text { Analysis of Maximum Likelihood Parameter Estimates }\end{array}$} \\
\hline Parameter & Estimate & $\begin{array}{l}\text { Standard } \\
\text { Error }\end{array}$ & $\begin{array}{l}\text { Wald Chi } \\
\text { Square }\end{array}$ & $\mathrm{p}$-value \\
\hline Intercept & 0.3457 & 0.7686 & 0.20 & 0.6528 \\
\hline High Jerk Percentage & 0.1552 & 0.0474 & 10.70 & 0.0011 \\
\hline Curve & -0.0268 & 0.2163 & 0.02 & 0.9015 \\
\hline Weaving & -0.0506 & 0.2883 & 0.03 & 0.8606 \\
\hline Average Daily Traffic & 3.3271 & 2.4189 & 1.89 & 0.169 \\
\hline Dispersion & 0.2657 & 0.0944 & & \\
\hline \multicolumn{5}{|c|}{$\begin{array}{l}\text { Quarter Mile Segment Model } 4 \\
\text { Negative Binomial Regression Model with High Jerk and ADT } \\
\text { AIC (Akaike Information Criterion): } 232.98 \\
\text { Analysis of Maximum Likelihood Parameter Estimates }\end{array}$} \\
\hline Parameter & Estimate & $\begin{array}{l}\text { Standard } \\
\text { Error }\end{array}$ & $\begin{array}{l}\text { Wald Chi } \\
\text { Square }\end{array}$ & $\mathrm{p}$-value \\
\hline Intercept & 0.0508 & 0.8491 & 0.00 & 0.9523 \\
\hline High Jerk Percentage & 0.0974 & 0.0327 & 8.87 & 0.0029 \\
\hline Average Daily Traffic & 3.0514 & 2.3457 & 1.69 & 0.1933 \\
\hline Dispersion & 0.2959 & 0.1000 & & \\
\hline
\end{tabular}


As seen from Model 1, when the percentage of high jerk observations is used as the only explanatory variable for total crashes the resulting $p$-value from the negative binomial model suggests that it has a significant relationship with total observed crashes from the ten year period. P-values from Model 2 reveal that other factors of consideration do not yield a significant correlation with total observed crashes. Model 3 revealed that when all four factors are considered, the percentage of high jerk events still remains significant while the other factors remain insignificant. Finally, Model 4 showed that the high jerk percentage remains significant even when roadway exposure (in the form of ADT) is considered.

Inherently, this high jerk percentage should be a good measurement for crash potential and the results of this analysis help demonstrate this. It is particularly interesting to see that the high jerk percentage had a significant correlation while ADT did not, which further suggests that this method may be more efficient compared to use other traditional crash frequency analysis. To aid in understanding why this significant correlation existed, Figure 13 below shows the data in the form of linear heat graphs for total historical crashes per segment and two key independent variables, high jerk percentage and ADT. The plots in Figure 13 are broken into five separate heat categories based on the percentile of observations. From Figure 13 it can be seen that the high percentage of jerk observations generally tend to follow the trends in the crash while ADT values do not. 


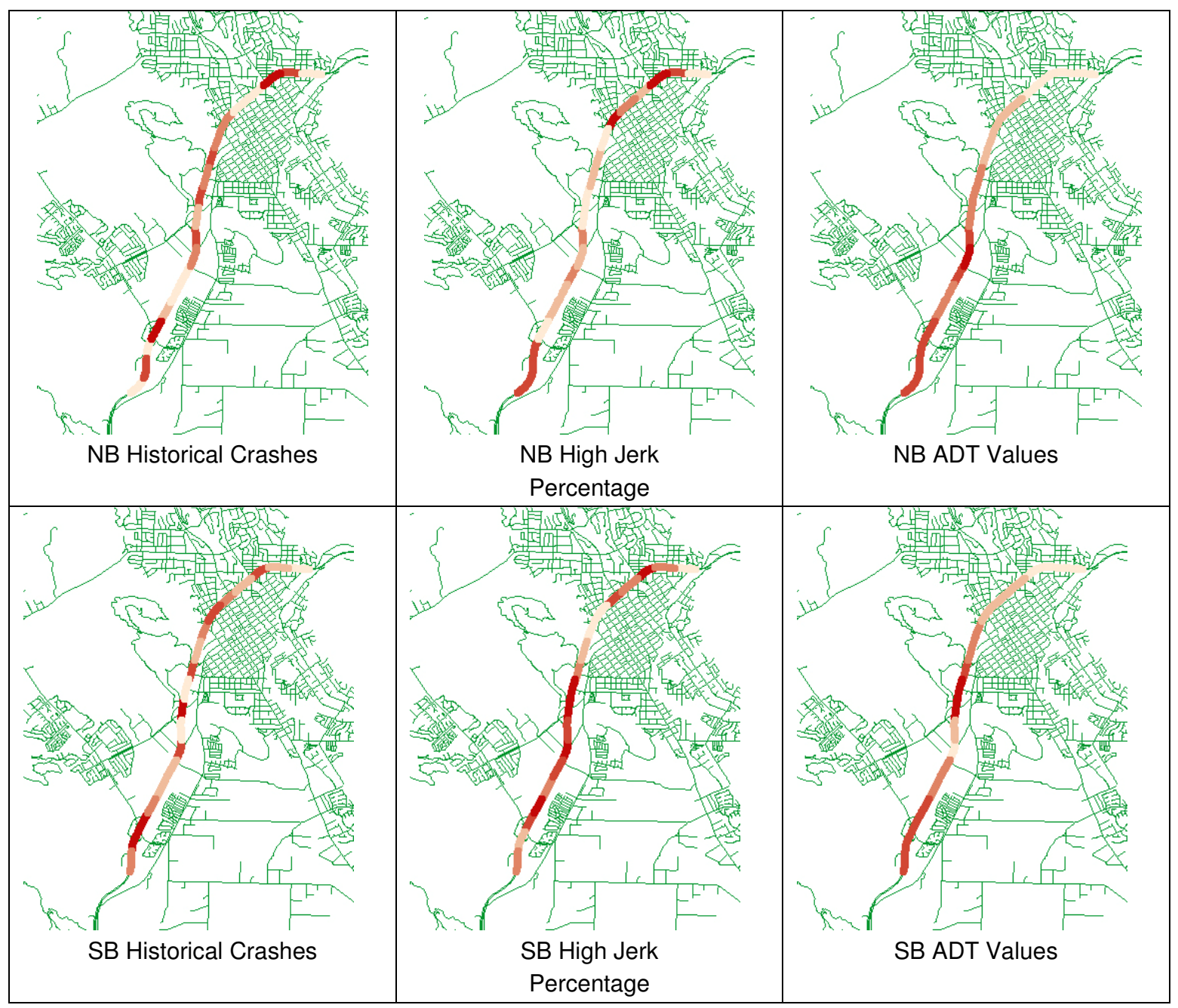

Figure 13. Linear Heat Maps for Quarter Mile Analysis

\section{HALF MILE ANALYSIS}

Analysis was repeated utilizing $1 / 2$-mile long segments to verify that trends observed in the first analysis remained true with larger analysis segment. 1/2-mile segments were constructed by combining two $1 / 4$-mile segments. A total of $191 / 2-$ mile long segments were constructed from the first 38 1/4-mile long segment. From these 19 segments, 10 are from the NB direction of US Highway 101, and 9 of them are from the SB direction of US Highway 101. Figure 14 shows the straight line diagrams for these 19 1/2-mile long segments. 

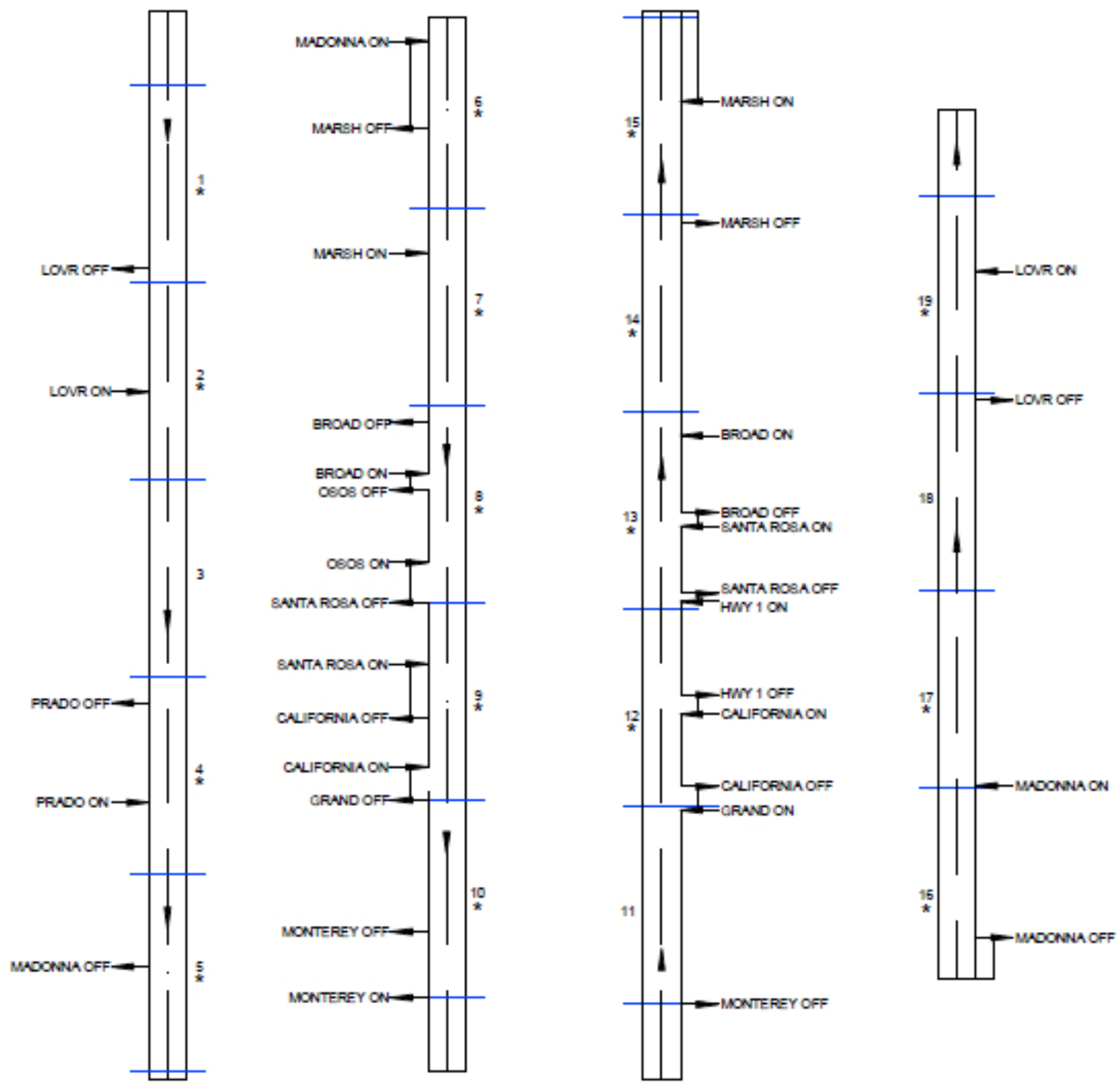

Figure 14. Straight line diagrams for US NB (left, 1-10) and SB (right, 11-20) half-mile segments

The same three negative binomial regression models were computed using the 1/2-mile long analysis segments. Table 7 shows the results of each negative binomial model with p-value results that were significant at a 95\% confidence level highlighted. 


\section{Table 7. Crash Estimation utilizing Negative Binomial Regression Models for Half Mile Segments}

\begin{tabular}{|c|c|c|c|c|}
\hline \multicolumn{5}{|c|}{$\begin{array}{l}\text { Half Mile Segment Model } 1 \\
\text { Negative Binomial Regression Model with High Jerk } \\
\text { AIC (Akaike Information Criterion): } 125.16 \\
\text { Analysis of Maximum Likelihood Parameter Estimates }\end{array}$} \\
\hline Parameter & Estimate & $\begin{array}{l}\text { Standard } \\
\text { Error }\end{array}$ & $\begin{array}{l}\text { Wald Chi } \\
\text { Square }\end{array}$ & p-value \\
\hline Intercept & 1.6340 & 0.4899 & 11.12 & .0009 \\
\hline High Jerk Percentage & 0.0944 & 0.0410 & 5.31 & 0.0212 \\
\hline Dispersion & 0.0795 & 0.0457 & & \\
\hline \multicolumn{5}{|c|}{$\begin{array}{l}\text { Half Mile Segment Model } 2 \\
\text { Negative Binomial Regression Model with ADT and Geometric Variables } \\
\text { AIC (Akaike Information Criterion): } 127.50 \\
\text { Analysis of Maximum Likelihood Parameter Estimates }\end{array}$} \\
\hline Parameter & Estimate & $\begin{array}{l}\text { Standard } \\
\text { Error }\end{array}$ & $\begin{array}{l}\text { Wald Chi } \\
\text { Square }\end{array}$ & p-value \\
\hline Intercept & 1.9638 & 0.6291 & 9.74 & 0.0018 \\
\hline Curve & 0.5724 & 0.2906 & 3.88 & 0.0489 \\
\hline Weaving & 0.2136 & 0.2189 & 0.95 & 0.3292 \\
\hline Average Daily Traffic & 0.7622 & 2.2424 & 0.12 & 0.7339 \\
\hline Dispersion & 0.0759 & 0.0421 & & \\
\hline \multicolumn{5}{|c|}{$\begin{array}{l}\text { Half Mile Segment Model } 3 \\
\text { Negative Binomial Regression Model with High Jerk, ADT and Geometric Variables } \\
\text { AIC (Akaike Information Criterion): } 123.90 \\
\text { Analysis of Maximum Likelihood Parameter Estimates }\end{array}$} \\
\hline Parameter & Estimate & $\begin{array}{l}\text { Standard } \\
\text { Error }\end{array}$ & $\begin{array}{l}\text { Wald Chi } \\
\text { Square }\end{array}$ & $\mathrm{p}$-value \\
\hline Intercept & 0.6163 & 0.7729 & 0.64 & 0.4252 \\
\hline High Jerk Percentage & 0.0929 & 0.0363 & 6.55 & 0.0105 \\
\hline Curve & 0.5084 & 0.2626 & 3.75 & 0.0529 \\
\hline Weaving & 0.1925 & 0.1908 & 1.02 & 0.3130 \\
\hline Average Daily Traffic & 1.9171 & 2.0402 & 0.88 & 0.3474 \\
\hline Dispersion & 0.0412 & 0.0315 & & \\
\hline \multicolumn{5}{|c|}{$\begin{array}{l}\text { Half Mile Segment Model } 4 \\
\text { Negative Binomial Regression Model with High Jerk and ADT } \\
\text { AIC (Akaike Information Criterion): } 126.46 \\
\text { Analysis of Maximum Likelihood Parameter Estimates }\end{array}$} \\
\hline Parameter & Estimate & $\begin{array}{l}\text { Standard } \\
\text { Error }\end{array}$ & $\begin{array}{l}\text { Wald Chi } \\
\text { Square }\end{array}$ & $p$-value \\
\hline Intercept & 1.0643 & 0.8253 & 1.66 & 0.1972 \\
\hline High Jerk Percentage & 0.1041 & 0.0416 & 6.25 & 0.0124 \\
\hline Average Daily Traffic & 1.6744 & 1.9802 & 0.71 & 0.3978 \\
\hline Dispersion & 0.0746 & 0.0440 & & \\
\hline
\end{tabular}


Results of the three models for $1 / 2$-mile segments were similar to the results seen in the $1 / 4$-mile segment analysis. Model 1 for the $1 / 2$-mile long segments shows that the high jerk percentage has a significant relationship with total crashes observed. This fact holds true in Model 3 and Model 4 as well. However, Model 2 showed that the feature "curve", which is a binary variable assigned to the segment if a curve is present in the segment, appears to be significant as well. This variable was not significant in the $1 / 4$-mile analysis. However, when observing the data it can be noted that there were only 3 out of the total 19 considered $1 / 2$-mile segments that did not contain curvature. This lack of sampling size for non-curve sections resulted in a very high standard error in model coefficient. Because of this high standard error it is believed that this significant correlation with curvature is a function of the small sample and may not be a reliable indicator of crash frequency.

Heat charts showing relative values of total historical crashes, high jerk percentages, and ADT values for the each bin are shown in Figure 15. Like the plots from in the $1 / 4$-mile analyses, the trends in the high jerk percentage follow the trends in crash data more closely than the ADT. 


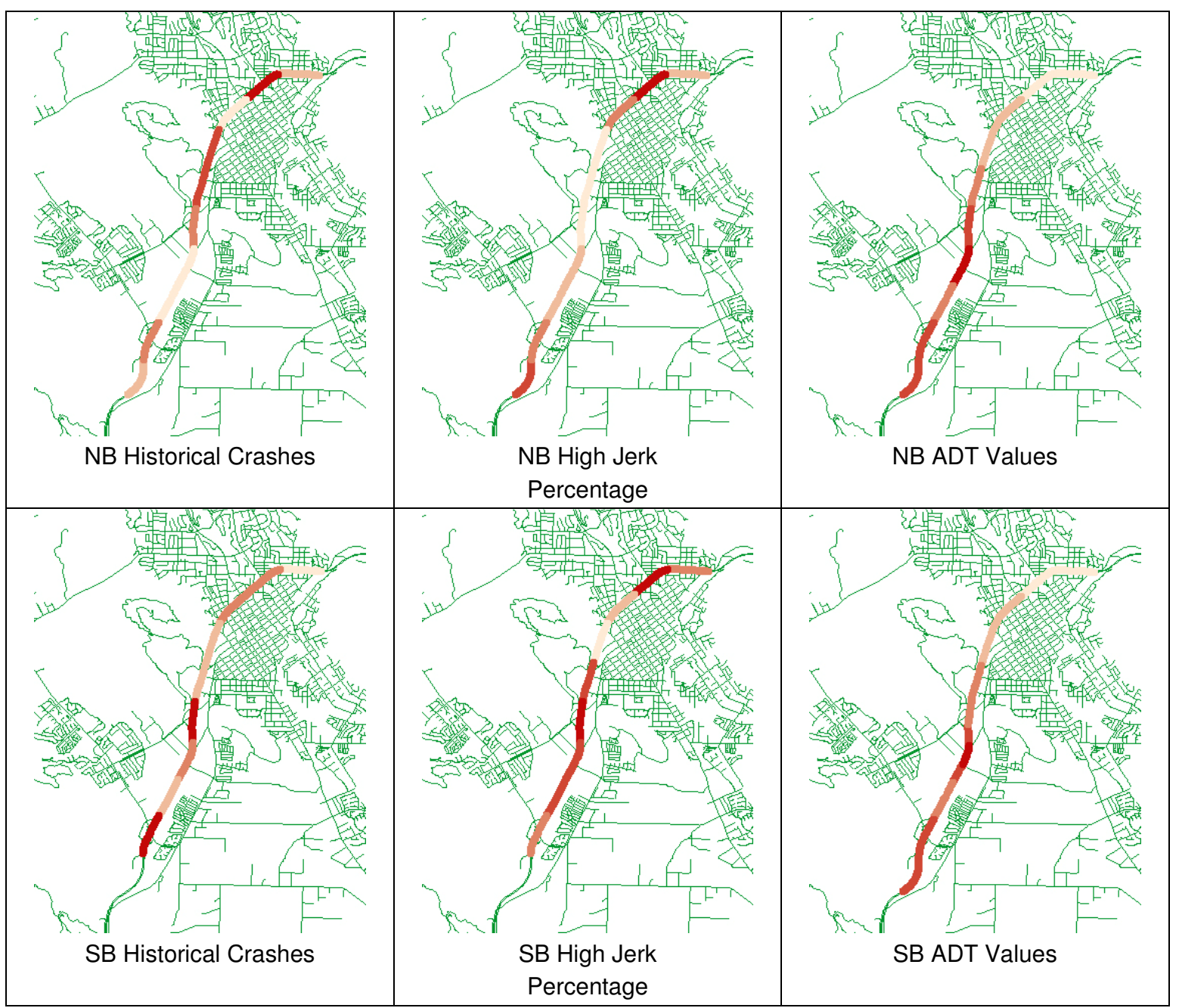

Figure 15. Linear Heat Maps for Half Mile Analysis

\section{CONCLUDING REMARKS}

The conclusion that high GPS jerk observation percentage has a strong correlation with historic crashes (i.e. long-term safety performance of the highway) and may indicate a possible connection to traffic safety analysis that was not previous explored. While the results of this analysis are strong, there is a need for more data to verify that these results are obtainable for multiple highways. This particular analysis was restricted to this one highway based on the nature of the natural- 
istic driving data collected. It is also interesting to see from this analysis that both the $1 / 4-$ mile and $1 / 2$-mile long segments showed this significant relationship of crashes and high jerk percentage. This suggests that this analysis can be valuable with different segment lengths traditionally used in crash frequency analysis. 


\section{CONCLUSIONS}

The objective of this research was to investigate the relationship between information obtained from naturalistic GPS driving data and long term safety performance on various types of roadway facilities. GPS driving data was collected as part of a naturalistic driving study from 33 Cal Poly staff members using data loggers from July 2012 to March 2013. Strict data processing and linear referencing performed in ESRI ArcMAP matched GPS data points to roadway attributes and ensured that no erroneous data was utilized for analysis.

Overall, the GPS data loggers performed considerably well under a variety of conditions and were able to determine vehicle movement with reasonable accuracy. This research demonstrates the value of this data through the use of two

analysis techniques for different types of roadway facilities. For arterial roadway analysis, Two-Fluid parameters for two local arterial roadways were obtained from GPS data. For highway segments, the GPS data was used to look at percentages of large negative jerk values in correlation with historical traffic crashes. Specific results related to each of the two major analysis methods are provided below, in addition to a discussion of potential future work.

\section{TWO-FLUID ANALYSIS FOR ARTERIAL ROADWAYS}

For analysis performed for arterial roadways, the Two-Fluid model was determined to be an appropriate model to assess long-term traffic safety performance based on previous literature (Dixit et al., 2011). Using linearly referenced GPS data, total travel time and stop time were found for individual trips on two arterial roadways near Cal Poly, Grand Avenue and California Boulevard. From this data 
the Two-Fluid model parameters could be obtained.

With a stop threshold of $2 \mathrm{MPH}$, a large portion of trips observed did not contain any time for which the vehicle was stopped. This fact is interesting considering both study areas included controlled intersections. A portion of these data points with no stop time were with trips in which the participant may have performed a rolling stop maneuver (undetected by the GPS data logger). It is more likely, that a large cluster may have resulted from the GPS noise resulting from inaccurate speed readings at lower travel speeds.

The Two-Fluid model parameter $n$, which represented a measure to the resistance of the roadway to degrade operation with increased demand, was determined to be 2.81 for Grand Avenue and .794 for California Boulevard. The parameter $T_{m}$, which represented the average minimum trip time for each $1 / 2$-mile analysis segment, was found to be 1.02 minutes for Grand Avenue and 1.11 minutes for California Boulevard. Based on previous research by Dixit et al. (2011) these Two-Fluid parameters would suggest that Grand Avenue is more susceptible to a higher crash rates.

Two-Fluid data points collected from the GPS data along California Boulevard were verified using data collected using the traditional "car chase" methodology. Car chase data was collected during the same peak periods during which a majority of the GPS data trips were observed. By using a general linear model procedure, the two data sources were proven to have no significance difference with trips that involved higher stop time. 


\section{HIGHWAY ANALYSIS}

The observed percentage of 'high' jerk data readings for highway segments was shown to be significantly correlated with historical crash rates for the segments in the preliminary analysis. Similar results were obtained for $1 / 4$-mile long segments and $1 / 2$-mile long segments along US Highway 101 . The correlations were significant at a 95\% confidence level. These correlations were also illustrated using heat maps to show that the percentage of high jerk readings tended to follow general trends observed in crash counts.

In this analysis it was interesting to see that the high jerk percentage performed better as an explanatory variable for total crashes when compared to three other factors including the presence of curve, presence of auxiliary lane, and ADT. This is best seen in the $1 / 4$-mile analysis, where high jerk percentage is the only factor proven with a significant relationship to traffic crash counts. While the binary attribute of curvature appeared to be significantly related with crash counts on the 1/2-mile segments, the coefficient estimate were not be reliable.

While the results of this analysis show strong correlation, the need for more data and further research to verify these trends is necessary. If this methodology is verified with additional data, the high jerk percentage measurement could act as a indicator of crash potential for highway segments. Other factors, such as segment length, must also be further considered to ensure the trends observed are of value. 


\section{FUTURE WORK}

The prospect of utilizing GPS driving data for a variety of transportation studies is a growing opportunity. Many mobile electronic devices, including many smartphones, contain GPS receivers that could possibly collect and store data similar to that employed in this research. This could permit "crowd sourcing" techniques for collecting data through the use of a cellular application. Utilizing crowd sourcing techniques can make data collection easier and more cost effective compared to other naturalistic driving studies.

Advancements in GPS system design are increasing this prospect as well. The removal of selective availability (SA) and the new L2C signal has significantly in-

creased the possible precision that GPS receivers can triangulate to. The speed performance of GPS receivers has considerably increased over the past years, which also allows more detailed analysis of vehicle trajectory. Other new GPS technologies such as real time kinematic (RTK) GPS receivers, which can provide significantly more accurate GPS data, will continue to become more commercially available for personal electronic devices.

Despite these prospects, there are still many hurtles that would need to be cleared before large scale GPS driving data collection could become a reality for agencies. The major limitation is privacy, which still remains a major concern to many individuals in the United States. The privacy concerns can be partially alleviated if private commercial fleet data was used. In addition to the hurtles related to privacy, Implementing data collection over a variety of different devices would also require careful detail to data collection, and would likely require electronic 
devices to rely solely on SPS instead of assisted GPS (aGPS) systems which many devices currently use.

\section{Improvements to this Research}

The major limitation of this research (as with many naturalistic driving studies) is the relatively small sample size. Increasing the total number of participants would strengthen the analysis performed on the roadways in this study and in future research. Further work relating this data with other data collected from Louisiana State University (LSU) will help in this regard, and will allow for comparisons between roadways. In addition to sample size, the Two-Fluid portion of analysis would be improved by further investigating the "stop" threshold to see if changes in the threshold value significantly impact Two-Fluid model parameters obtained.

\section{Additional Topics of Study}

While it was not covered in this research, it would be interesting to further segregate GPS driving data in various categories (i.e. based on time of day, participant information, etc.). Relating to the highway analysis, it would also be fascinating to further study the locations of high jerk GPS readings in relation to locations of certain geometric features (i.e. curvature, signage, etc.) to see if a higher frequency of high jerk occur in response to certain defined features. 


\section{ABBREVIATIONS AND ACRONYMS}

ADT: Average Daily Traffic

aGPS: Assisted Global Positioning System

Cal Poly: California Polytechnic State University, San Luis Obispo

DAS: Data Acquisition System

Data Logger: OHARARP SD GPS Data Loggers V3.15

DOP: Dilution of Precision

GPS: Global Positioning System

GIS: Geographic Information Systems

LSU: Louisiana State University

MOE: Measure of Effectiveness

NB: Northbound

NMEA: National Marine Electronics Association

RTK: Real-Time Kinematic

SA: Selective Availability

SAS: Statistic Analysis Software

SB: Southbound

SHRP: Strategic Highway Research Program 2

SPS: Standard Positioning Service

UTC: Coordinated Universal Time

WGS84: World Geodetic System 1984 Update 


\section{BIBLIOGRAPHY}

Abdel-Aty, Mohamed, and Essam Radwan. "Modeling Traffic Accident Occurence and Involvement." Accident Analysis \& Prevention 32.5 (2000): 633-642. Print.

Ardekani, S. "The Two-Fluid Characterization of Urban Traffic: Theory, Observation, and Experiment." University of Texas at Austin,1984. Print.

Ardekani, S, and Robert Herman. "Urban Network-Wide Variables and Their Relations." Transportation Science 21.1 (1987): 1-16. Print.

Ardekani, S, V Torres-Verdin, and Robert Herman. "The Two-Fluid Model and Traffic Quality in Mexico City (El Modelo Bifluido y La Calidad Del Transito En La Ciudad de Mexico)." Revista Ingenieria Civil (1985). Print.

Bagdadi, Omar. "Assessing Safety Critical Braking Events in Naturalistic Driving Studies." Transportation Research Part F: Traffic Psychology and Behaviour 16 (2013): 117-126. ScienceDirect. Web. 13 Aug. 2013.

Bagdadi, Omar, and András Várhelyi. "Development of a Method for Detecting Jerks in Safety Critical Events." Accident Analysis \& Prevention 50 (2013): 83-91. ScienceDirect. Web. 22 July 2013.

Campbell, Ken. "SHRP 2 Analysis Planning." Second International Symposium on Naturalistic Driving Research. (2010).

Dixit, Vinayak V et al. "Quality of Traffic Flow on Urban Arterial Streets and Its Relationship with Safety." Accident; analysis and prevention 43.5 (2011): 16101616. NCBI PubMed. Web.

Guo, Feng et al. Evaluating the Relationship Between Near-Crashes and Crashes: Can Near-Crashes Serve as a Surrogate Safety Metric for Crashes? National Highway Traffic Safety Administration, 2010. Print.

Hauer, Ezra. "Identification of Sites with Promise." Transportation Research Record: Journal of the Transportation Research Board 1542 (1996): 54-60. Print.

Herman, Robert, and Prigogine llya. "A Two-Fluid Approach to Town Traffic." Science 204.4389 (1979): 148-151. Print.

---. "Kinetic Theory of Vehicular Traffic" American Elsevier (1971): 440-452. Print.

Herman, Robert, L Malakhoff, and S Ardekani. "Trip Time-Stop Time Studies of Extreme Driver Behaviors." Transportation Research Part A: General 6.26 (1988): 427-433. Print. 
Hong, Sang Yeon et al. "Analysis of Two-Fluid Model Using GPS Data." Journal of the Eastern Asia Society for Transportation Studies 6 (2005): 560-572. Print.

Jones, Elizabeth, and Farhat Wahid. "Validation of Two-Fluid Model of Urban Traffic for Arterial Streets." Transportation Research Record: Journal of the Transportation Research Board 1876.1 (2004): 132-141. Print.

Klauer, Shella et al. The Impact of Driver Inattention on Near-Crash/Crash Risk: An Analysis Using the 100-Car Naturalistic Driving Study Data. National Highway Traffic Safety Administration, 2006. Print.

Lee, Chungwon et al. "Characterizing Urban Network Performance Using Two-Fluid Model." Journal of the Eastern Asia Society for Transportation Studies 6 (2005): 1534-1544. Print.

McCracken, Heather, and Derek Law. "Linear Referencing: An Introduction." 2008 ESRI User Conference. San Diego Convention Center. 2008. Lecture.

Milton, J, and F Mannering. "The Relationship Among Highway Geometrics, Trafficrelated Elements and Motor-vehicle Accident Frequencies." Transportation 25.4 (1998): 395-413. Print.

Neale, Vicki et al. "An Overview of the 100-Car Naturalistic Study and Findings." Washington D.C., 2005. Print.

“Traffic Data Branch." California Department of Transportation.

“Transportation Injury Mapping System (TIMS)." University of California, Berkeley.

Vo, P et al. "Comparison of Quality of Service in Two Cenral Business Districts: TwoFluid Model Approach in Texas." Transportation Research Record: Journal of the Transportation Research Board 1999 (2007): 180-188. Print.

Williams, James. "Macroscopic Flow Models." Traffic Flow Theory. Oak Ridge National Laboratory, 1997. Print.

Williams, James, H Mahmassani, and Robert Herman. "Analysis of Traffic Network Flow Relations and Two-Fluid Model Parameter Sensitivity." Transportation Research Record: Journal of the Transportation Research Board 1005 (1985): 95106. Print. 
APPENDIX A: SAMPLE GPS OUTPUT

\begin{tabular}{|c|c|c|c|c|c|c|c|c|c|c|c|c|c|c|c|c|}
\hline LATITUDE & LONGITUDE & ALTITUDE & HEADING & SPEED & SAT & PDOP & HDOP & VDOP & $\mathrm{FIX}$ & YEAR & MONTH & DAY & HOUR & MIN & SEC & MSEC \\
\hline 35.25122834 & \begin{tabular}{|l}
-120.6784439 \\
\end{tabular} & 32.7 & 206.38 & 64.4 & 9 & 1.139 & 0.85 & 0.77 & 1 & 2013 & 2 & 15 & 15 & 23 & 2 & 100 \\
\hline \begin{tabular}{|l|}
35.25113678 \\
\end{tabular} & -120.678505 & 32.59 & 206.39 & 64.39 & 9 & 1.139 & 0.85 & 0.77 & 1 & 2013 & 2 & 15 & 15 & 23 & 2 & 500 \\
\hline 35.25102234 & \begin{tabular}{|l|l}
-120.6785736 \\
\end{tabular} & 32.59 & 206.52 & 64.31 & 9 & 1.139 & 0.85 & 0.77 & 1 & 2013 & 2 & 15 & 15 & 23 & 3 & 0 \\
\hline 35.25092698 & -120.678627 & 32.59 & 206.38 & 64.23 & 9 & 1.139 & 0.85 & 0.77 & 1 & 2013 & 2 & 15 & 15 & 23 & 3 & 400 \\
\hline 35.25083542 & \begin{tabular}{|l|l|}
-120.6786881 \\
\end{tabular} & 32.59 & 206.19 & 64.25 & 9 & 1.48 & 0.85 & 1.21 & 1 & 2013 & 2 & 15 & 15 & 23 & 3 & 800 \\
\hline \begin{tabular}{|l|}
35.25074006 \\
\end{tabular} & \begin{tabular}{|l}
-120.6787414 \\
\end{tabular} & 32.5 & 206.11 & 64.17 & 9 & 1.139 & 0.85 & 0.77 & 1 & 2013 & 2 & 15 & 15 & 23 & 4 & 200 \\
\hline 35.2506485 & \begin{tabular}{|l}
-120.6788025 \\
\end{tabular} & 32.5 & 206.08 & 64.09 & 9 & 1.139 & 0.85 & 0.77 & 1 & 2013 & 2 & 15 & 15 & 23 & 4 & 600 \\
\hline 35.25055314 & -120.6788559 & 32.4 & 206.11 & 64.09 & 9 & 1.139 & 0.85 & 0.77 & 1 & 2013 & 2 & 15 & 15 & 23 & 5 & 0 \\
\hline \begin{tabular}{|l|}
35.25046158 \\
\end{tabular} & -120.678917 & 32.4 & 206.19 & 64.06 & 9 & 1.47 & 0.85 & 1.2 & 1 & 2013 & 2 & 15 & 15 & 23 & 5 & 400 \\
\hline 35.25037002 & \begin{tabular}{|l|l}
-120.6789703 \\
\end{tabular} & 32.29 & 206.17 & 64.31 & 9 & 1.47 & 0.85 & 1.2 & 1 & 2013 & 2 & 15 & 15 & 23 & 5 & 800 \\
\hline \begin{tabular}{|l|}
35.25027848 \\
\end{tabular} & -120.6790238 & 32.29 & 206.13 & 64.29 & 9 & 1.47 & 0.85 & 1.2 & 1 & 2013 & 2 & 15 & 15 & 23 & 6 & 200 \\
\hline 35.2501831 & \begin{tabular}{|l|l|}
-120.6790848 \\
\end{tabular} & 32.2 & 206.27 & 64.25 & 10 & 1.419 & 0.8 & 1.169 & 1 & 2013 & 2 & 15 & 15 & 23 & 6 & 600 \\
\hline \begin{tabular}{|l|l|}
35.25008774 \\
\end{tabular} & \begin{tabular}{|l|l|l}
-120.6792 \\
\end{tabular} & 32.29 & 206.19 & 64.09 & 9 & 1.47 & 0.85 & 1.2 & 1 & 2013 & 2 & 15 & 15 & 23 & 7 & 0 \\
\hline \begin{tabular}{|l|}
55.24999618 \\
\end{tabular} & \begin{tabular}{|l|l|}
-120.6791992 \\
\end{tabular} & 32.2 & 206.28 & 64.02 & 9 & 1.47 & 0.85 & 1.2 & 1 & 2013 & 2 & 15 & 15 & 23 & 7 & 400 \\
\hline \begin{tabular}{|l|}
35.24990082 \\
\end{tabular} & \begin{tabular}{|l}
-120.6792526 \\
\end{tabular} & 32.2 & 206.3 & 64.17 & 9 & 1.47 & 0.85 & 1.2 & 1 & 2013 & 2 & 15 & 15 & 23 & 7 & 800 \\
\hline \begin{tabular}{|l|}
55.24980926 \\
\end{tabular} & -120.679306 & 32.2 & 206.41 & 64.22 & 10 & 1.419 & 0.8 & 1.169 & 1 & 2013 & 2 & 15 & 15 & 23 & 8 & 200 \\
\hline 35.2497139 & -120.679367 & 32.2 & 206.38 & 64.07 & 10 & 1.1 & 0.8 & 0.76 & 1 & 2013 & 2 & 15 & 15 & 23 & 8 & 600 \\
\hline 35.24962234 & \begin{tabular}{|l|}
-120.6794205 \\
\end{tabular} & 32.09 & 206.41 & 64.08 & 9 & 1.139 & 0.85 & 0.77 & 1 & 2013 & 2 & 15 & 15 & 23 & 9 & 0 \\
\hline 35.2495308 & \begin{tabular}{|l|}
-120.6794738 \\
\end{tabular} & 32.09 & 206.36 & 64.05 & 9 & 1.139 & 0.85 & 0.77 & 1 & 2013 & 2 & 15 & 15 & 23 & 9 & 400 \\
\hline 35.24943542 & \begin{tabular}{|l}
-120.6795349 \\
\end{tabular} & 32 & 206.47 & 63.97 & 9 & 1.139 & 0.85 & 0.77 & 1 & 2013 & 2 & 15 & 15 & 23 & 9 & 800 \\
\hline 35.24934388 & \begin{tabular}{|l}
-120.6795883 \\
\end{tabular} & 31.89 & 206.71 & 63.94 & 9 & 1.139 & 0.85 & 0.77 & 1 & 2013 & 2 & 15 & 15 & 23 & 10 & 200 \\
\hline 35.24925232 & \begin{tabular}{|l}
-120.6796494 \\
\end{tabular} & 31.89 & 206.88 & 63.69 & 9 & 1.139 & 0.85 & 0.77 & 1 & 2013 & 2 & 15 & 15 & 23 & 10 & 600 \\
\hline \begin{tabular}{|l|}
35.24916076 \\
\end{tabular} & \begin{tabular}{|l|l|l}
-120.677 \\
\end{tabular} & 31.79 & 206.99 & 63.43 & 9 & 1.139 & 0.85 & 0.77 & 1 & 2013 & 2 & 15 & 15 & 23 & 11 & 0 \\
\hline \begin{tabular}{|l}
35.24907302 \\
\end{tabular} & -120.6797562 & 31.7 & 207.03 & 63.15 & 9 & 1.139 & 0.85 & 0.77 & 1 & 2013 & 2 & 15 & 15 & 23 & 11 & 400 \\
\hline 35.24898148 & \begin{tabular}{|l}
-120.6798172 \\
\end{tabular} & 31.7 & 207.14 & 62.76 & 9 & 1.139 & 0.85 & 0.77 & 1 & 2013 & 2 & 15 & 15 & 23 & 11 & 800 \\
\hline 35.24889374 & \begin{tabular}{|l|l|l}
-120.679806 \\
\end{tabular} & 31.6 & 207.25 & 62.31 & 9 & 1.139 & 0.85 & 0.77 & 1 & 2013 & 2 & 15 & 15 & 23 & 12 & 200 \\
\hline 35.248806 & -120.679924 & 31.5 & 207.35 & 61.77 & 9 & 1.139 & 0.85 & 0.77 & 1 & 2013 & 2 & 15 & 15 & 23 & 12 & 600 \\
\hline 35.24871826 & \begin{tabular}{|l}
-120.6799774 \\
\end{tabular} & 31.5 & 207.57 & 61.49 & 9 & 1.139 & 0.85 & 0.77 & 1 & 2013 & 2 & 15 & 15 & 23 & 13 & 0 \\
\hline 35.24863052 & -120.6800308 & 31.39 & 207.82 & 61.1 & 9 & 1.139 & 0.85 & 0.77 & 1 & 2013 & 2 & 15 & 15 & 23 & 13 & 400 \\
\hline 35.2485466 & \begin{tabular}{|l}
-120.6800918 \\
\end{tabular} & 31.29 & 208 & 60.81 & 9 & 1.139 & 0.85 & 0.77 & 1 & 2013 & 2 & 15 & 15 & 23 & 13 & 800 \\
\hline 35.24845886 & -120.6801453 & 31.2 & 207.91 & 60.3 & 9 & 1.139 & 0.85 & 0.77 & 1 & 2013 & 2 & 15 & 15 & 23 & 14 & 200 \\
\hline 35.24837494 & -120.6801986 & 31.1 & 207.77 & 59.79 & 9 & 1.139 & 0.85 & 0.77 & 1 & 2013 & 2 & 15 & 15 & 23 & 14 & 600 \\
\hline 35.24829102 & -120.6802521 & 31 & 207.63 & 59.26 & 9 & 1.139 & 0.85 & 0.77 & 1 & 2013 & 2 & 15 & 15 & 23 & 15 & 0 \\
\hline 35.2482071 & -120.6803055 & 30.89 & 207.42 & 58.74 & 9 & 1.139 & 0.85 & 0.77 & 1 & 2013 & 2 & 15 & 15 & 23 & 15 & 400 \\
\hline \begin{tabular}{|l|}
35.24812698 \\
\end{tabular} & \begin{tabular}{|l}
-120.6803513 \\
\end{tabular} & 30.79 & 207.27 & 58.2 & 9 & 1.139 & 0.85 & 0.77 & 1 & 2013 & 2 & 15 & 15 & 23 & 15 & 800 \\
\hline 35.24804688 & -120.6804046 & 30.7 & 207.21 & 57.59 & 9 & 1.139 & 0.85 & 0.77 & 1 & 2013 & 2 & 15 & 15 & 23 & 16 & 200 \\
\hline 35.24796676 & \begin{tabular}{|l}
-120.6804504 \\
\end{tabular} & 30.5 & 207.07 & 57.07 & 9 & 1.139 & 0.85 & 0.77 & 1 & 2013 & 2 & 15 & 15 & 23 & 16 & 600 \\
\hline 35.24788284 & -120.6805038 & 30.39 & 206.86 & 56.71 & 9 & 1.139 & 0.85 & 0.77 & 1 & 2013 & 2 & 15 & 15 & 23 & 17 & 0 \\
\hline 35.24780274 & \begin{tabular}{|l}
-120.6805496 \\
\end{tabular} & 30.29 & 206.75 & 56.06 & 9 & 1.139 & 0.85 & 0.77 & 1 & 2013 & 2 & 15 & 15 & 23 & 17 & 400 \\
\hline 35.24772644 & -120.6805954 & 30.2 & 206.77 & 55.38 & 9 & 1.139 & 0.85 & 0.77 & 1 & 2013 & 2 & 15 & 15 & 23 & 17 & 800 \\
\hline 35.24764634 & \begin{tabular}{|l|l|}
-120.680688 \\
\end{tabular} & 30.1 & 207.08 & 54.76 & 9 & 1.139 & 0.85 & 0.77 & 1 & 2013 & 2 & 15 & 15 & 23 & 18 & 200 \\
\hline 35.24757004 & \begin{tabular}{|l}
-120.6806946 \\
\end{tabular} & 30 & 207.36 & 54.02 & 9 & 1.139 & 0.85 & 0.77 & 1 & 2013 & 2 & 15 & 15 & 23 & 18 & 600 \\
\hline 35.24749756 & \begin{tabular}{|l}
-120.6807403 \\
\end{tabular} & 29.89 & 207.8 & 53.25 & 9 & 1.139 & 0.85 & 0.77 & 1 & 2013 & 2 & 15 & 15 & 23 & 19 & 0 \\
\hline 35.24742126 & $\mid-120.6807862$ & 29.79 & 208.17 & 52.83 & 9 & 1.139 & 0.85 & 0.77 & 1 & 2013 & 2 & 15 & 15 & 23 & 19 & 400 \\
\hline 35.24734878 & -120.6808395 & 29.7 & 208.44 & 52.3 & 9 & 1.139 & 0.85 & 0.77 & 1 & 2013 & 2 & 15 & 15 & 23 & 19 & 800 \\
\hline 35.2472763 & -120.6808853 & 29.6 & 208.75 & 51.75 & 9 & 1.139 & 0.85 & 0.77 & 1 & 2013 & 2 & 15 & 15 & 23 & 20 & 200 \\
\hline 35.24720382 & -120.6809311 & 29.5 & 209.02 & 51.07 & 9 & 1.139 & 0.85 & 0.77 & 1 & 2013 & 2 & 15 & 15 & 23 & 20 & 600 \\
\hline 35.24713516 & -120.6809845 & 29.39 & 208.97 & 50.07 & 9 & 1.139 & 0.85 & 0.77 & 1 & 2013 & 2 & 15 & 15 & 23 & 21 & 0 \\
\hline 35.24707032 & -120.6810302 & 29.29 & 209.03 & 49.31 & 9 & 1.139 & 0.85 & 0.77 & 1 & 2013 & 2 & 15 & 15 & 23 & 21 & 400 \\
\hline 35.24700164 & $\mid-120.6810761$ & 29.2 & 209.3 & 48.53 & 9 & 1.139 & 0.85 & 0.77 & 1 & 2013 & 2 & 15 & 15 & 23 & 21 & 800 \\
\hline 35.24693298 & \begin{tabular}{|l|}
-120.6811218 \\
\end{tabular} & 29 & 209.58 & 47.76 & 9 & 1.139 & 0.85 & 0.77 & 1 & 2013 & 2 & 15 & 15 & 23 & 22 & 200 \\
\hline 35.24687194 & -120.6811676 & 28.89 & 209.8 & 46.87 & 10 & 1.1 & 0.8 & 0.76 & 1 & 2013 & 2 & 15 & 15 & 23 & 22 & 600 \\
\hline 35.2468071 & -120.6812058 & 28.79 & 209.97 & 45.78 & 10 & 1.1 & 0.8 & 0.76 & 1 & 2013 & 2 & 15 & 15 & 23 & 23 & 0 \\
\hline 35.24674606 & \begin{tabular}{|l}
-120.6812515 \\
\end{tabular} & 28.7 & 210.36 & 45.09 & 10 & 1.1 & 0.8 & 0.76 & 1 & 2013 & 2 & 15 & 15 & 23 & 23 & 400 \\
\hline 35.24668502 & \begin{tabular}{|l}
-120.6812973 \\
\end{tabular} & 28.6 & 210.86 & 44.42 & 10 & 1.1 & 0.8 & 0.76 & 1 & 2013 & 2 & 15 & 15 & 23 & 23 & 800 \\
\hline 35.24663162 & -120.681343 & 28.39 & 211.74 & 43.78 & 10 & 1.1 & 0.8 & 0.76 & 1 & 2013 & 2 & 15 & 15 & 23 & 24 & 200 \\
\hline 35.2465744 & -120.6813889 & 28.2 & 213.05 & 43.1 & 10 & 1.419 & 0.8 & 1.169 & 1 & 2013 & 2 & 15 & 15 & 23 & 24 & 600 \\
\hline 35.24652482 & \begin{tabular}{|l}
-120.6814346 \\
\end{tabular} & 28.1 & 215.41 & 42.31 & 10 & 1.419 & 0.8 & 1.169 & 1 & 2013 & 2 & 15 & 15 & 23 & 25 & 0 \\
\hline 35.24647522 & \begin{tabular}{|l|l|}
-120.6804 \\
\end{tabular} & 28 & 217.58 & 41.3 & 10 & 1.1 & 0.8 & 0.76 & 1 & 2013 & 2 & 15 & 15 & 23 & 25 & 400 \\
\hline 35.24642944 & \begin{tabular}{|l}
-120.6815338 \\
\end{tabular} & 27.79 & 220.11 & 40.61 & 10 & 1.1 & 0.8 & 0.76 & 1 & 2013 & 2 & 15 & 15 & 23 & 25 & 800 \\
\hline 35.24638366 & \begin{tabular}{|l}
-120.6815872 \\
\end{tabular} & 27.6 & 223.02 & 40.03 & 10 & 1.419 & 0.8 & 1.169 & 1 & 2013 & 2 & 15 & 15 & 23 & 26 & 200 \\
\hline 35.2463417 & \begin{tabular}{|l}
-120.6816406 \\
\end{tabular} & 27.5 & 226.13 & 39.78 & 10 & 1.1 & 0.8 & 0.76 & 1 & 2013 & 2 & 15 & 15 & 23 & 26 & 600 \\
\hline 35.24630356 & -120.681694 & 27.39 & 228.52 & 38.72 & 10 & 1.1 & 0.8 & 0.76 & 1 & 2013 & 2 & 15 & 15 & 23 & 27 & 0 \\
\hline 35.24626542 & -120.681755 & 27.29 & 231.63 & 38.09 & 10 & 1.1 & 0.8 & 0.76 & 1 & 2013 & 2 & 15 & 15 & 23 & 27 & 400 \\
\hline 35.24623108 & \begin{tabular}{|l|l|}
-120.6818161 \\
\end{tabular} & 27.2 & 233.57 & 37.48 & 10 & 1.419 & 0.8 & 1.169 & 1 & 2013 & 2 & 15 & 15 & 23 & 27 & 800 \\
\hline 35.24620056 & -120.6818695 & 27.1 & 235.58 & 36.07 & 10 & 1.1 & 0.8 & 0.76 & 1 & 2013 & 2 & 15 & 15 & 23 & 28 & 200 \\
\hline 35.24617386 & \begin{tabular}{|l}
-120.6819306 \\
\end{tabular} & 27 & 237.44 & 34.82 & 10 & 1.1 & 0.8 & 0.76 & 1 & 2013 & 2 & 15 & 15 & 23 & 28 & 600 \\
\hline 35.24614334 & -120.6819992 & 26.89 & 240.13 & 33.56 & 10 & 1.1 & 0.8 & 0.76 & 1 & 2013 & 2 & 15 & 15 & 23 & 29 & 100 \\
\hline 35.24612046 & \begin{tabular}{|l}
-120.6820602 \\
\end{tabular} & 26.89 & 241.96 & 32.4 & 10 & 1.1 & 0.8 & 0.76 & 1 & 2013 & 2 & 15 & 15 & 23 & 29 & 500 \\
\hline 35.24610138 & -120.6821137 & 26.79 & 243.44 & 31.19 & 10 & 1.1 & 0.8 & 0.76 & 1 & 2013 & 2 & 15 & 15 & 23 & 29 & 900 \\
\hline
\end{tabular}




\section{APPENDIX B: SAMPLE SAS CODE FOR DATA VALIDATION}

/*Import California GPS and Verification Data*/

data test;

input Int Intr verify;

datalines;

4.6249728134 .2195077051

4.7184988714 .2195077051

4.4426512564 .3174881141

5.1590552994 .2484952421

5.1647859744 .7874917431

4.6634390944 .3694478521

4.6728288344 .3694478521

4.499809674 .2904594411

4.8441870864 .499809671

4.7791234934 .2904594411

4.4067192474 .2195077051

4.4308167994 .1896547421

5.0689042024 .2341065051

5.1119877884 .5108595071

4.653960354 .3174881141

4.6347289884 .3174881141

4.3567088274 .1588830831

4.6821312274 .2484952421

4.5747109794 .330733341

4.4659081194 .2484952421

5.2311086174 .3694478521 
4.6151205174 .3438054221

4.6347289884 .3944491551

4.7273878194 .3174881141

4.7184988714 .3944491551

4.7874917434 .2766661191

4.8362819074 .2626798771

4.6347289884 .2626798771

4.5747109794 .3040650931

4.3944491554 .2766661191

5.0625950334 .4067192471

$4.17438727 \quad 4.0775374441$

4.3944491554 .1896547421

4.7184988714 .3438054221

4.3567088274 .174387271

4.7621739354 .2766661191

4.9126548864 .330733341

4.5325994934 .2766661191

4.0943445624 .0253516911

4.6249728134 .2626798771

4.3438054224 .2341065051

4.3820266354 .2046926191

4.3694478524 .1896547421

4.6347289884 .2904594411

4.4426512564 .2046926191

4.4773368144 .1896547421

4.4188406084 .2341065051 
4.6249728134 .3694478521

4.4659081194 .2626798771

5.1416635574 .5217885771

4.4773368144 .1271343851

4.3438054224 .2046926191

4.5108595074 .3040650931

4.6728288344 .2904594411

4.3694478524 .1431347261

4.7874917434 .3174881141

4.499809674 .1896547421

4.4188406084 .2484952421

4.3174881144 .1431347261

4.8520302644 .2626798771

4.4773368144 .2766661191

4.6728288344 .2195077051

$4.4426512564 .17438727 \quad 1$

4.3820266354 .1431347261

4.5432947824 .2046926191

4.6913478824 .3438054221

4.595119854 .3040650931

4.5643481914 .2626798771

4.3567088274 .2195077051

4.9558270584 .5849674791

4.3944491554 .2484952421

4.595119854 .4067192471

4.7273878194 .2046926191 
4.499809674 .3820266351

4.7621739354 .2341065051

4.5643481914 .2484952421

4.6249728134 .330733341

4.6443908994 .1431347261

4.4543472964 .2626798771

4.5747109794 .1896547421

4.5325994934 .2904594411

4.5325994934 .2484952421

4.595119854 .330733341

4.499809674 .2766661191

4.7361984484 .2626798771

4.7874917434 .1431347261

4.330733344 .1588830831

4.3567088274 .1431347261

4.3438054224 .0943445621

4.5108595074 .3567088271

4.4308167994 .2484952421

4.7361984484 .2046926191

5.1149953094 .7774414070

3.9759363313 .9759363310

4.8895969664 .4830025520

4.2398868684 .2398868680

4.1075897894 .1075897890

4.867534454 .5653893160

4.7510006344 .2986450260 
4.3040650934 .3040650930

4.5152454784 .4127982930

4.4426512564 .4379342670

4.4331949214 .2002049530

4.1206618714 .1206618710

$4.80073697 \quad 4.130355 \quad 0$

3.8958936233 .8958936230

$4.7723781054 .48187197 \quad 0$

4.9437829874 .0826093060

4.2959239364 .1956970560

4.5849674794 .2986450260

4.1399550734 .1399550730

4.3346729384 .2180360350

4.2341065054 .2341065050

4.8473317434 .675628650

4.6071681894 .2738844760

4.4284330074 .3907385750

4.3515674274 .3515674270

4.7587492744 .2355547310

4.6977493674 .2031989670

4.2180360354 .2180360350

4.9294252394 .3228072750

4.6530075154 .1573193610

$4.49980967 \quad 4.2918283670$

4.4682043314 .3618239270

4.2427645674 .2253728250 
4.3438054224 .0775374440

4.3528552574 .3174881140

4.8097423524 .5674683190

4.6453519764 .5580785780

4.6111522584 .1666652240

4.3027128284 .0656020930

4.3795235044 .3795235040

5.1010851974 .4284330070

4.3993752734 .3993752730

4.3359826964 .2106450180

4.8386600294 .7613188680

4.9045337634 .4975849750

4.6577626364 .6269316780

4.5890408044 .5293684730

4.640537334 .0535225680

4.4473461014 .4473461010

4.5808774934 .5042442670

4.3719762994 .3719762990

4.5981455714 .4115854370

3.9473901493 .9473901490

4.6337576434 .5736795190

4.8667649244 .5829245770

4.4379342674 .4379342670

4.4115854374 .4115854370

4.3707128754 .3707128750

4.4426512564 .4426512560 


\subsection{4 .4520190060 \\ 4.7388265714 .5580785780 \\ 4.7361984484 .5829245770 \\ 4.4704952834 .4659081190 \\ 4.7440621854 .5890408040 \\ 5.0795392734 .5075573570 \\ run;}

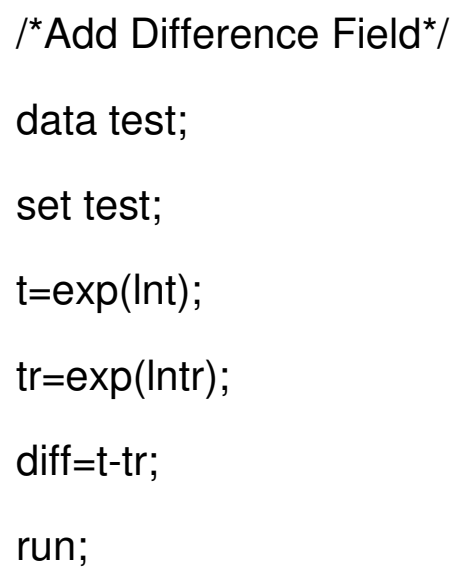




\title{
APPENDIX C: SAMPLE SAS CODE FOR HIGHWAY ANALYSIS
}

\author{
$/{ }^{*}$ Create Meas2 Field ${ }^{*} /$ \\ data thesis.Corrected101nb; \\ set thesis.Corrected101nb; \\ meas2 $=($ meas -84313.44$) / 5280$; \\ run; \\ data thesis.Corrected101sb; \\ set thesis.Corrected101sb; \\ meas $2=$ meas $/ 5280$; \\ run;
}

${ }^{*}$ Assign Jerk Variables to $\mathrm{NB}^{\star} /$

data thesis.Corrected101nb;

set thesis.Corrected101nb;

if (jerk $<-0.5$ and acc $<0$ ) then jerk0_1=1; else jerk0_1=0;

run;

data thesis.Corrected101nb;

set thesis.Corrected101nb; 
if (jerk $<0$ and acc $<0$ ) then jerk00_1=1; else jerk00_1=0;

run;

data thesis.Corrected101nb;

set thesis.Corrected101nb;

if (jerk $<-0.75$ and acc $<0$ ) then jerk075_1=1; else jerk075_1=0;

run;

data thesis.Corrected101nb;

set thesis.Corrected101nb;

if $($ jerk $<-1.0$ and acc $<0)$ then jerk1_1=1; else jerk1_1=0;

run;

data thesis.Corrected101nb;

set thesis.Corrected101nb;

if (jerk $<-1.25$ and acc $<0$ ) then jerk2_1=1; else jerk2_1=0;

run;

data thesis.Corrected101nb;

set thesis.Corrected101nb;

if (jerk $<-1.5$ and acc $<0$ ) then jerk3_1=1; else jerk3_1=0;

run; 
data thesis.Corrected101nb;

set thesis.Corrected101nb;

if (jerk $<-1.75$ and acc $<0$ )then jerk4_1 $1=1$; else jerk4_1=0;

run;

data thesis.Corrected101nb;

set thesis.Corrected101nb;

if $($ jerk $<-2.0$ and acc $<0)$ then jerk5_1=1; else jerk5_1=0;

run;

data thesis.Corrected101nb;

set thesis.Corrected101nb;

if (jerk $<-2.25$ and acc $<0)$ then jerk6_1=1; else jerk6_1=0;

run;

data thesis.Corrected101nb;

set thesis.Corrected101nb;

if (jerk $<-2.5$ and acc $<0)$ then jerk7_1=1; else jerk7_1=0;

run;

data thesis.Corrected101nb;

set thesis.Corrected101nb; 
if (jerk $<-2.75$ and acc $<0$ )then jerk8_1=1; else jerk8_1=0;

run;

data thesis.Corrected101nb;

set thesis.Corrected101nb;

if (jerk $<-3.0$ and acc $<0$ )then jerk9_1 $1=1$; else jerk9_1=0;

run;

data thesis.Corrected101nb;

set thesis.Corrected101nb;

if (jerk $<-3.0$ and acc $<0$ )then jerk9_1=1; else jerk9_1=0;

run;

data thesis.Corrected101nb;

set thesis.Corrected101nb;

if (jerk $<-3.5$ and acc $<0)$ then jerk10_1=1; else jerk10_1=0;

run;

data thesis.Corrected101nb;

set thesis.Corrected101nb;

if (jerk $<-4$ and acc $<$ ) then jerk11_1=1; else jerk11_1=0;

run; 
${ }^{*}$ Assign Jerk Variables to $\mathrm{SB}^{*} /$

data thesis.Corrected101sb;

set thesis.Corrected101sb;

if (jerk $<-0.5$ and acc $<0)$ then jerk0_1=1; else jerk0_1=0;

run;

data thesis.Corrected101sb;

set thesis.Corrected101sb;

if (jerk $<0$ and acc $<0)$ then jerk00_1=1; else jerk00_1=0;

run;

data thesis.Corrected101sb;

set thesis.Corrected101sb;

if (jerk $<-0.75$ and acc $<0$ ) then jerk075_1=1; else jerk075_1=0;

run;

data thesis.Corrected101sb;

set thesis.Corrected101sb;

if $($ jerk $<-1.0$ and acc $<0)$ then jerk1_1=1; else jerk1_1=0;

run; 
data thesis.Corrected101sb;

set thesis.Corrected101sb;

if (jerk $<-1.25$ and acc $<0$ ) then jerk2_1=1; else jerk2_1=0;

run;

data thesis.Corrected101sb;

set thesis.Corrected101sb;

if (jerk $<-1.5$ and acc $<0)$ then jerk3_1=1; else jerk3 $\_1=0$;

run;

data thesis.Corrected101sb;

set thesis.Corrected101sb;

if (jerk $<-1.75$ and acc $<0$ )then jerk4_1=1; else jerk4_1=0;

run;

data thesis.Corrected101sb;

set thesis.Corrected101sb;

if (jerk $<-2.0$ and acc $<0$ ) then jerk5_1=1; else jerk5_1=0;

run;

data thesis.Corrected101sb;

set thesis.Corrected101sb; 
if (jerk $<-2.25$ and acc $<0)$ then jerk6_1=1; else jerk6_1=0;

run;

data thesis.Corrected101sb;

set thesis.Corrected101sb;

if (jerk $<-2.5$ and acc $<$ ) then jerk7_1=1; else jerk7_1=0;

run;

data thesis.Corrected101sb;

set thesis.Corrected101sb;

if (jerk $<-2.75$ and acc $<0)$ then jerk8_1=1; else jerk8_1=0;

run;

data thesis.Corrected101sb;

set thesis.Corrected101sb;

if (jerk $<-3.0$ and acc $<0)$ then jerk9_1=1; else jerk9_1=0;

run;

data thesis.Corrected101nb;

set thesis.Corrected101nb;

if (jerk $<-3.0$ and acc $<0)$ then jerk9_1=1; else jerk9_1=0;

run; 
data thesis.Corrected101sb;

set thesis.Corrected101sb;

if (jerk $<-3.5$ and acc $<0)$ then jerk10_1=1; else jerk10_1=0;

run;

data thesis.Corrected101sb;

set thesis.Corrected101sb;

if (jerk $<-4$ and acc $<0)$ then jerk11_1=1; else jerk11_1=0;

run;

$/^{*}$ Macro Run, repeat for each threshold level ${ }^{\star} /$

\%macro datacheck1(heading1, heading2, headingp,headingm, mp1, mp2, jerkN,oput,seg);

proc freq data=thesis.Corrected $101 \mathrm{nb}$;

where $\quad(\& m p 1<$ meas2 $<\& m p 2 \quad$ and $\quad$ (\&heading1\&headingm) $<=$ heading $<=$ (\&heading2+\&headingp) and $\mathrm{HDOP}<=3$ and delta_T>0);

tables \&jerkN /out=\&oput;

run;

data \&oput; 
set \&oput;

segment=\&seg;

run;

\%mend datacheck1;

\%macro datacheck2(heading1, heading2,headingp,headingm, heading3, mp1, mp2, jerkN,oput,seg);

proc freq data=thesis. Corrected $101 \mathrm{nb}$;

where (\&mp1<meas2<\&mp2 and (heading<=(\&heading2+\&headingp) or heading $>=$ \&heading3) and $\mathrm{HDOP}<=3$ and delta_T $>0$ );

tables \&jerkN /out=\&oput;

run;

data \&oput;

set \&oput;

segment=\&seg;

run;

\%mend datacheck2;

TITLE 'NEW DATA';

\%datacheck1(26,54,3,3,33.81,34.06,jerk8_1,j8_1,1);

\%datacheck2(0,26,3,3,357,34.06,34.31,jerk8_1,j8_2,2); 
\%datacheck2(0,26,3,3,357,34.31,34.56,jerk8_1,j8_3,3);

\%datacheck1(26,26,3,3,34.56,34.81,jerk8_1,j8_4,4);

\%datacheck1(26,26,3,3,34.81,35.06,jerk8_1,j8_5,5);

\%datacheck1(26,26,3,3,35.06,35.31,jerk8_1,j8_6,6);

\%datacheck1(26,26,3,3,35.31,35.56,jerk8_1,j8_7,7);

\%datacheck1(10,26,3,3,35.56,35.81,jerk8_1,j8_8,8);

\%datacheck2(0,10,3,3,353,35.81,36.06,jerk8_1,j8_9,9);

\%datacheck2(0,2,3,3,353,36.06,36.31,jerk8_1,j8_10,10);

\%datacheck1(2,15,3,3,36.31,36.56,jerk8_1,j8_11,11);

\%datacheck1(15,15,3,3,36.56,36.81,jerk8_1,j8_12,12);

\%datacheck1(15,15,3,3,36.81,37.06,jerk8_1,j8_13,13);

\%datacheck1(15,26,3,3,37.06,37.31,jerk8_1,j8_14,14);

\%datacheck1(26,43,3,3,37.31,37.56,jerk8_1,j8_15,15);

\%datacheck1(43,48,3,3,37.56,37.81,jerk8_1,j8_16,16);

\%datacheck1(48,48,3,3,37.81,38.06,jerk8_1,j8_17,17);

\%datacheck1(48,75,3,3,38.06,38.31,jerk8_1,j8_18,18);

\%datacheck1(75,92,3,3,38.31,38.56,jerk8_1,j8_19,19);

\%datacheck1(92,92,3,3,38.56,38.81,jerk8_1,j8_20,20); 
\%macro datacheck3(heading1, heading2, headingp,headingm, mp1, mp2, jerkN,oput,seg);

proc freq data=thesis. Corrected $101 \mathrm{sb}$;

where $\quad(\& m p 1<$ meas2<\&mp2 and $\quad$ (\&heading1\&headingm $)<=$ heading $<=$ (\&heading $2+$ \&headingp) and $\mathrm{HDOP}<=3$ and delta_T>0);

tables \&jerkN /out=\&oput;

run;

data \&oput;

set \&oput;

segment=\&seg;

run;

$\%$ mend datacheck3;

TITLE 'NEW DATA';

\%datacheck3(272,272,3,3,41.0216121590907,41.2716121590907,jerk8_1,j8_21, 21);

\%datacheck3(272,272,3,3,41.2716121590907,41.5216121590907,jerk8_1,j8_22, 22);

\%datacheck3(233,272,3,3,41.5216121590907,41.7716121590907,jerk8_1,j8_23, 23); 
\%datacheck3(228,233,3,3,41.7716121590907,42.0216121590907,jerk8_1,j8_24, 24);

\%datacheck3(228,228,3,3,42.0216121590907,42.2716121590907,jerk8_1,j8_25, 25);

\%datacheck3(205,228,3,3,42.2716121590907,42.5216121590907,jerk8_1,j8_26, 26);

\%datacheck3(200,205,3,3,42.5216121590907,42.7716121590907,jerk8_1,j8_27, 27);

\%datacheck3(195,200,3,3,42.7716121590907,43.0216121590907,jerk8_1,j8_28, 28);

\%datacheck3(195,195,3,3,43.0216121590907,43.2716121590907,jerk8_1,j8_29, 29);

\%datacheck3(186,195,3,3,43.2716121590907,43.5216121590907,jerk8_1,j8_30, 30);

\%datacheck3(186,186,3,3,43.5216121590907,43.7716121590907,jerk8_1,j8_31, $31)$

\%datacheck3(176,186,3,3,43.7716121590907,44.0216121590907,jerk8_1,j8_32, 32);

\%datacheck3(176,198,3,3,44.0216121590907,44.2716121590907,jerk8_1,j8_33, 33);

\%datacheck3(198,206,3,3,44.2716121590907,44.5216121590907,jerk8_1,j8_34, 82 
34);

\%datacheck3(206,206,3,3,44.5216121590907,44.7716121590907,jerk8_1,j8_35, $35)$;

\%datacheck3(206,206,3,3,44.7716121590907,45.0216121590907,jerk8_1,j8_36, $36)$;

\%datacheck3(206,206,3,3,45.0216121590907,45.2716121590907,jerk8_1,j8_37, 37 ;

\%datacheck3(182,206,3,3,45.2716121590907,45.5216121590907,jerk8_1,j8_38, 38);

\%datacheck3(182,185,3,3,45.5216121590907,45.7716121590907,jerk8_1,j8_39, $39)$;

$/{ }^{*}$ Create Combined Proc Freq Tables, repeat for each jerk threshold level ${ }^{*} /$

data work.j11_final;

set j11_1-j11_39;

run;

/*Assign Curve and Weaving Data*/

data thesis.Finaldata_7_30_13;

set thesis.Finaldata_7_30_13; 


\begin{tabular}{|c|c|c|}
\hline if segment $1=1$ & then curve= & 1 \\
\hline if segment $1=2$ & then curve $=$ & 1 \\
\hline if segment $1=3$ & then curve $=$ & 1 \\
\hline if segment $1=4$ & then curve $=$ & 0 \\
\hline if segment $1=5$ & then curve $=$ & 0 \\
\hline if segment $1=6$ & then curve $=$ & 0 \\
\hline if segment $1=7$ & then curve $=$ & 0 \\
\hline if segment $1=8$ & then curve $=$ & 1 \\
\hline if segment $1=9$ & then curve $=$ & 1 \\
\hline if segment $1=10$ & then curve $=$ & 1 \\
\hline if segment $1=11$ & then curve $=$ & 1 \\
\hline if segment $1=12$ & then curve $=$ & 0 \\
\hline if segment $1=13$ & then curve $=$ & 0 \\
\hline if segment $1=14$ & then curve $=$ & 1 \\
\hline if segment $1=15$ & then curve $=$ & 1 \\
\hline if segment $1=16$ & then curve $=$ & 1 \\
\hline if segment $1=17$ & then curve $=$ & 0 \\
\hline$=1$ & urve $=$ & \\
\hline
\end{tabular}




\begin{tabular}{|c|c|c|}
\hline if segment $1=19$ & then curve $=$ & 1 \\
\hline if segment $1=20$ & then curve= & 0 \\
\hline if segment $1=21$ & then curve= & 0 \\
\hline if segment $1=22$ & then curve= & 0 \\
\hline if segment $1=23$ & then curve $=$ & 1 \\
\hline if segment $1=24$ & then curve $=$ & 1 \\
\hline if segment $1=25$ & then curve $=$ & 0 \\
\hline if segment $1=26$ & then curve $=$ & 1 \\
\hline if segment $1=27$ & then curve= & 1 \\
\hline if segment $1=28$ & then curve $=$ & 1 \\
\hline if segment $1=29$ & then curve $=$ & 0 \\
\hline if segment $1=30$ & then curve $=$ & 1 \\
\hline if segment $1=31$ & then curve $=$ & 0 \\
\hline if segment $1=32$ & then curve $=$ & 1 \\
\hline if segment $1=33$ & then curve= & 1 \\
\hline if segment $1=34$ & then curve $=$ & 1 \\
\hline if segment $1=35$ & then curve $=$ & 0 \\
\hline seg & then curve $=$ & 0 \\
\hline
\end{tabular}




if segment $1=37 \quad$ then curve $=0$
if segment $1=38 \quad$ then curve $=1 ;$
if segment $1=39 \quad$ then curve $=1 ;$

run;

data thesis.Finaldata_7_30_13;

set thesis.Finaldata_7_30_13;

if segment $1=1 \quad$ then weaving $=\quad 0 \quad$;

if segment $1=2 \quad$ then weaving $=0 ;$

if segment $1=3 \quad$ then weaving $=\quad 0 \quad$;

if segment $1=4 \quad$ then weaving $=0 ;$

if segment $1=5 \quad$ then weaving $=\quad 0 \quad$;

if segment $1=6 \quad$ then weaving $=\quad 0 \quad$;

if segment $1=7 \quad$ then weaving $=\quad 0 \quad ;$

if segment $1=8 \quad$ then weaving $=0 ;$

if segment $1=9 \quad$ then weaving $=\quad 0 \quad$;

if segment $1=10 \quad$ then weaving $=\quad 0 \quad$;

if segment $1=11 \quad$ then weaving $=\quad 1 ;$

if segment $1=12$ then weaving $=1$; 


\begin{tabular}{|c|c|c|}
\hline if segment $1=13$ & then weaving= & 0 \\
\hline if segment $1=14$ & then weaving= & 1 \\
\hline if segment $1=15$ & then weaving= & 1 \\
\hline if segment $1=16$ & then weaving= & 1 \\
\hline if segment $1=17$ & then weaving= & 1 \\
\hline if segment $1=18$ & then weaving= & 1 \\
\hline if segment $1=19$ & then weaving= & 0 \\
\hline if segment $1=20$ & then weaving= & 0 \\
\hline if segment $1=21$ & then weaving= & 0 \\
\hline if segment $1=22$ & then weaving= & 0 \\
\hline if segment $1=23$ & then weaving= & 1 \\
\hline if segment $1=24$ & then weaving= & 1 \\
\hline if segment $1=25$ & then weaving= & 1 \\
\hline if segment $1=26$ & then weaving= & 0 \\
\hline if segment $1=27$ & then weaving= & 0 \\
\hline if segment $1=28$ & then weaving= & 0 \\
\hline if segment $1=29$ & then weaving= & 0 \\
\hline if segment $1=30$ & then weaving= & ) \\
\hline
\end{tabular}




\begin{tabular}{|c|c|c|}
\hline if segment $1=31$ & then weaving= & 0 \\
\hline if segment $1=32$ & then weaving= & 0 \\
\hline if segment $1=33$ & then weaving= & 0 \\
\hline if segment $1=34$ & then weaving= & 0 \\
\hline if segment $1=35$ & then weaving= & 0 \\
\hline if segment $1=36$ & then weaving= & 0 \\
\hline if segment $1=37$ & then weaving= & 0 \\
\hline if segment $1=38$ & then weaving= & 0 \\
\hline if segment $1=39$ & then weaving= & \\
\hline
\end{tabular}

run;

/*Perform Pearson Correlation Coefficient Analysis, repeat for half mile*/

proc corr data=thesis.Finaldata_7_30_13

pearson

plots=matrix $($ histogram $)$;

*where segment $\sim=32$;

var set2rate j0pct j1pct j3pct j5pct j7pct j9pct j10pct j11pct;

run; 
/*Perform Chi Square Tests, repeat for half mile*/

/*Just Jerk*

proc genmod data=thesis.Finaldata_7_30_13r;

model set2_count $=$ j1pct_DOP $/$ dist=negbin;

run;

${ }^{*}$ Traditional Model ${ }^{*}$

proc genmod data=thesis.Finaldata_7_30_13;

model set2_count = curve weaving adt2 /dist=negbin;

run;

${ }^{*}$ Traditional Model with Jerk*/

proc genmod data=thesis.Finaldata_7_30_13;

model set2_count =j1pct_DOP curve weaving adt2 /dist=negbin;

run;

${ }^{*}$ Jerk and $\mathrm{ADT}^{*} /$

proc genmod data=thesis.Finaldata_7_30_13; 
model set2_count $=j 1$ pct_DOP adt2 /dist=negbin;

run;

/*Generate Standard Error Plot for Curves* $/$

DATA thesis.Finaldata_7_30_13;

SET thesis. Finaldata_7_30_13;

IF Curve=0 THEN Curve2='NO';

IF Curve=1 THEN Curve2='YES';

run;

PATTERN1 COLOR=GRAYCC;

AXIS1 LABEL=(F="ARIAL/BOLD" "J1PCT")

MINOR=NONE;

AXIS2 LABEL=(F="ARIAL/BOLD" "Curve");

TITLE1 'Mean With Error Bars, Version 2';

TITLE2 '(With 95\% Confidence Limits)';

PROC GCHART DATA=thesis.Finaldata_7_30_13;

VBAR Curve2 / 
WIDTH=15

TYPE=MEAN

INSIDE=MEAN

ERRORBAR=TOP

CLM=95

SUMVAR=J1PCT_DOP

RAXIS=AXIS1 MAXIS=AXIS2

COUTLINE=BLACK WOUTLINE $=1$;

RUN;

QUIT; 